\title{
Evaluation and refinement of sample preparation methods for extracellular matrix proteome coverage
}

\author{
Maxwell C. McCabet, , Lauren R. Schmitt+, , Ryan C. Hill ${ }^{1,2}$, Monika Dzieciatkowska,2, Mark \\ Maslanka ${ }^{1,2}$, Willeke F. Daamen ${ }^{3}$, Toin H. van Kuppevelt ${ }^{3}$, Danique J. Hof ${ }^{3}$, Kirk C. Hansen ${ }^{* 1,2}$
}

AFFILIATION.

${ }^{1}$ Department of Biochemistry and Molecular Genetics, School of Medicine, University of

Colorado, Aurora, CO, USA

${ }^{2}$ Cancer Center Proteomics Core, School of Medicine, University of Colorado, Aurora, CO, USA

${ }^{3}$ Department of Biochemistry, Radboud Institute for Molecular Life Sciences, Radboud University

Medical Center, Nijmegen, The Netherlands

†These authors contributed equally to this work.

*Corresponding author.

KEYWORDS. extracellular matrix, proteomics, sample preparation methods, collagen, glycoprotein, proteoglycan, matrisome 


\section{ABSTRACT}

The extracellular matrix is a key component of tissues, yet it is under-represented in proteomic datasets. Identification and evaluation of proteins in the extracellular matrix (ECM) has proved challenging due to the insolubility of many ECM proteins in traditional protein extraction buffers. Here we separate the decellularization and ECM extraction steps of several prominent methods for evaluation under real-world conditions. The results are used to optimize a two-fraction ECM extraction method. Approximately one dozen additional parameters are tested and recommendations for analysis based on overall ECM coverage or specific ECM classes are given. Compared to a standard in-solution digest, the optimized method yielded a 4-fold improvement in unique ECM peptide identifications.
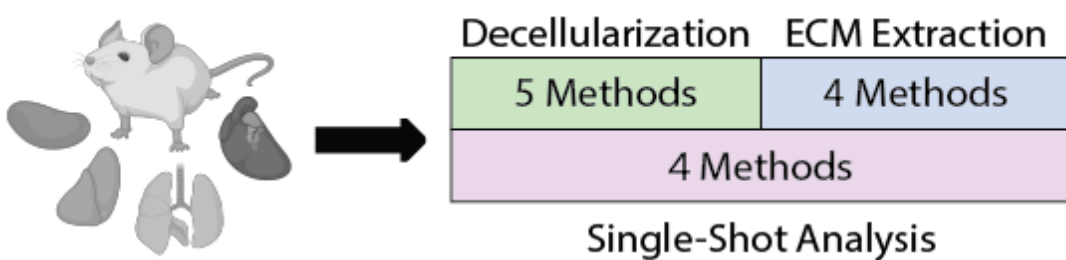

Single-Shot Analysis

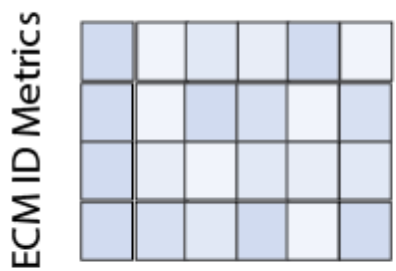

Method Variations

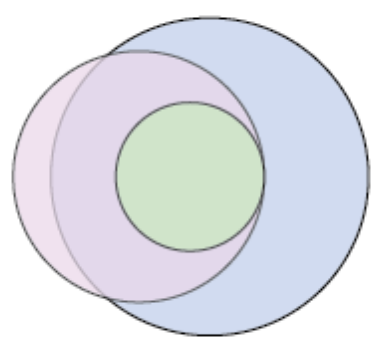

Evaluation of ECM Coverage

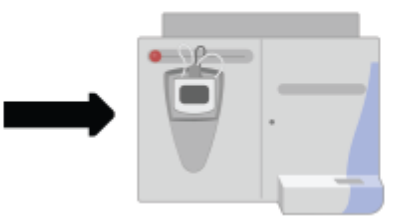

】

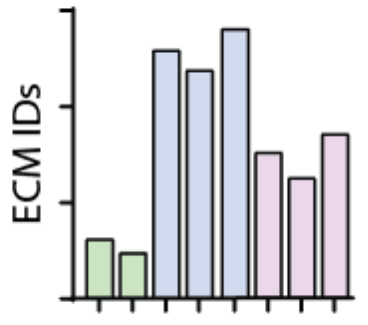

Methods 


\section{$\underline{\text { Introduction }}$}

The extracellular matrix (ECM) is a non-cellular component of tissues which provides structural scaffolding and mediates signaling in the extracellular space to govern a wide range of biological processes including cell differentiation, wound healing, and fibrosis. ${ }^{1}$ Knowledge of ECM composition is critical to fields ranging from biomedical to food science, yet data regarding the ECM proteome is relatively sparse. In general, proteins of the ECM assemble into, or interact with, extended non-covalent polymers. ${ }^{2}$ Several core structural proteins undergo post-translational modifications including crosslink generation to further stabilize the assembled structures, ${ }^{3}$ rendering this sizable covalent fraction resistant to extraction in the strongest detergents and chaotropic agents. ${ }^{4}$ Often, collagen-containing ECM structural fibers are quantified using assays measuring hydroxyproline as a surrogate for collagen after total protein hydrolysis. ${ }^{5}$ The results are a crude measurement of total collagen and provide no information about collagen subtype distribution or solubility. Additionally, hydroxyproline residues are present in other cellular and extracellular proteins such as HIF- $1 a^{6}$ and elastin ${ }^{7}$ which hinders the specificity of the assay. In other studies, second harmonic generation (SHG) and two-photon autofluorescence (TPAF) imaging have been successfully used to characterize ECM fibers by taking advantage of the intrinsic properties of collagen fibers and elastin autofluorescence to allow for analysis of ECM architecture. ${ }^{8,9}$ SHG and TPAF along with other imaging approaches can be used to determine the properties of ECM fibers and their degree of alignment and branching, yet they fail to provide specific qualitative and quantitative information about matrix protein abundance and subtype. 
Proteomics is an attractive approach to complement these methods due to its ability to provide both compositional and quantitative readouts. Preliminary draft proteomes have been reported in recent years with deep proteome coverage of tissues obtained from protein extraction in a strong chaotrope and extended LC-MS acquisition. ${ }^{10-13}$ However, ECM proteins that were expected to be highly abundant (e.g. collagens I, III, V, elastin, etc.) were not found at high abundance in these datasets. This is likely a result of approximately $75-85 \%$ of the fibrillar ECM residing in a chaotrope-resistant insoluble fraction that has eluded analysis by these and other standard proteomic methods. $^{14}$

Protein extraction protocols have been developed which specifically target enrichment of the ECM. These methods typically consist of a decellularization step followed by chaotrope extraction, either alone ${ }^{15-18}$ or followed by dilution and digestion with LysC and/or Trypsin in preparation for LC-MS/MS analysis. ${ }^{19-25}$ Biochemical methods have also been developed which use chemical digestion to solubilize and access highly insoluble ECM proteins of interest. ${ }^{4,26}$ We have previously developed methods which utilize chemical digestion with cyanogen bromide $(\mathrm{CNBr})^{14}$ and hydroxylamine hydrochloride $(\mathrm{HA}){ }^{4}$ While both methods efficiently extract insoluble proteins, HA digestion has been the method of choice due to its safety, low cost, and lack of additional transfer steps during processing. ${ }^{4}$ However, non-specific Asn-X cleavages should be considered during database searching and the digestion process can induce oxidative modifications that, if extended beyond methionine single oxidation, can convolute data analysis, leaving room for improvement of the method. ${ }^{4}$

A wide variety of decellularization and ECM extraction methods have been published but it remains unclear how these methods perform compared to one another on a complex, ECM-rich sample. Previous matrisome enrichment method comparisons have been performed ${ }^{27}$ revealing 
the strength of chemical digestion in identifying core matrisome (structural ECM) proteins. However, significant advancements and new methods have since been developed. A direct comparison of both cell and ECM extraction methods on a whole organism sample and four additional organs serves as an important reference for future ECM proteomics. Putative proteins which compose the ECM have been previously defined using in silico and proteomic approaches, generating the MatrisomeDB which is divided into core matrisome and matrisome-associated proteins. ${ }^{28}$ Here, we utilize core matrisome annotations of collagens, ECM glycoproteins, and proteoglycans for comparison of ECM protein characterization. For this comparison, five widely used decellularization methods ${ }^{4,19,29}$ and four methods for single-shot extraction and analysis $^{21,30,31}$ (Table 1) have been used for evaluation. In addition, four ECM extraction methods: a two-step extraction with guanidine hydrochloride followed by hydroxylamine hydrochloride (Gnd-HCl/HA) digestion, ${ }^{4}$ chaotrope-assisted in-solution digest with ultrasonication (CAISU), ${ }^{19}$ chaotrope-assisted in-solution digest (CAIS),${ }^{20}$ and surfactant and chaotropic agent assisted sequential extraction/on-pellet digestion (SCAD) ${ }^{21}$ were evaluated. The findings are used to make recommendations for tissue analysis based on factors including matrisome protein sequence coverage, the number of matrisome proteins identified, and variability of results.

\section{$\underline{\text { Methods }}$}

Table 1. Descriptions of tested decellularization, ECM extraction, and single shot methods with required time and composition of each extraction buffer. 


\begin{tabular}{|c|c|c|c|}
\hline Method & $\begin{array}{r}\text { Steps Involving } \\
\text { Buffer Exchange }\end{array}$ & Time & Buffer Composition \\
\hline 1-Fraction $\mathrm{A}^{4}$ & 3 & $3 \mathrm{hrs}$ & 1. $50 \mathrm{mM}$ Tris- $\mathrm{HCl}, 0.25 \%$ CHAPS, $25 \mathrm{mM}$ EDTA $3 \mathrm{M} \mathrm{NaCl}, 10 \mu \mathrm{L} / \mathrm{mL} \mathrm{PI}, \mathrm{pH} 7.4$ \\
\hline 1-Fraction $\mathrm{B}^{28}$ & 5 & 7 days & $\begin{array}{l}\text { 1. } 10 \mathrm{mM} \text { Tris- } \mathrm{HCl}, 0.1 \% \text { EDTA, } 1 \times \mathrm{PI}, \mathrm{pH} 8.0 \\
\text { 2. } 50 \mathrm{mM} \text { Tris- } \mathrm{HCl} \mathrm{pH} 7.4,10 \mathrm{mM} \mathrm{MgCl}, 1 \% \text { benzonase, } 1 \times \mathrm{PI}\end{array}$ \\
\hline 2-Fraction & 2 & 2 hrs & $\begin{array}{l}\text { 1. } 150 \mathrm{mM} \mathrm{NaCl}, 50 \mathrm{mM} \text { Tris- } \mathrm{HCl}, 5 \% \text { glycerol, } 1 \% \mathrm{NP}-40,1 \mathrm{mM} \mathrm{MgCl}, 1 \times \mathrm{PI} \\
\text { 2. } 50 \mathrm{mM} \text { Tris- } \mathrm{HCl} \mathrm{pH} 7.4,2 \% \text { CHAPS, } 2 \mathrm{mM} \text { EDTA, } 3 \mathrm{M} \mathrm{NaCl}, 1 \times \mathrm{PI}\end{array}$ \\
\hline 3-Fraction ${ }^{18}$ & 4 & $3 \mathrm{hrs}$ & $\begin{array}{l}\text { 1. } 150 \mathrm{mM} \mathrm{NaCl}, 50 \mathrm{mM} \text { Tris- } \mathrm{HCl} \text { pH } 7.5,5 \% \text { glycerol, } 1 \% \mathrm{NP}-40,1 \mathrm{mM} \mathrm{MgCl}, 1 \mathrm{x} \\
\mathrm{PI}, 1 \% \text { benzonase } \\
\text { 2. } 150 \mathrm{mM} \mathrm{NaCl}, 50 \mathrm{mM} \text { Tris-HCl pH } 7.5,5 \% \text { glycerol, } 1 \% \mathrm{NP}-40,0.5 \% \mathrm{DOC} \text {, } \\
\text { 0.1\% } \mathrm{SDS}, 1 \times \mathrm{PI}, 1 \% \text { benzonase } \\
\text { 3. } 500 \mathrm{mM} \mathrm{NaCl}, 50 \mathrm{mM} \text { Tris-HCl pH } 7.5,5 \% \text { glycerol, } 1 \% \mathrm{NP}-40,2 \% \mathrm{DOC}, 1 \% \\
\text { SDS, } 1 \times \mathrm{PI}, 1 \% \text { benzonase }\end{array}$ \\
\hline 4-Fraction & 10 & $8.5 \mathrm{hrs}$ & 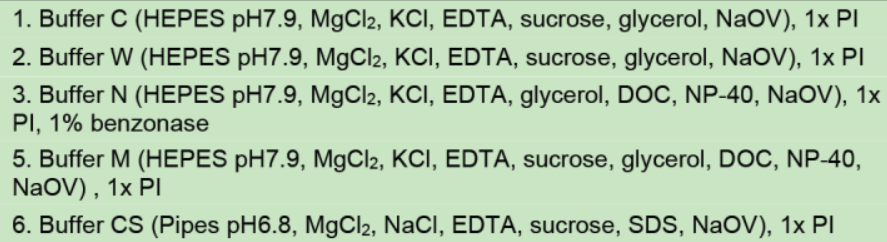 \\
\hline Gnd-HCl/HA ${ }^{4}$ & 3 & 2.5 days & $\begin{array}{l}\text { 1. } 6 \mathrm{M} \mathrm{Gnd}-\mathrm{HCl}, 100 \mathrm{mM} \mathrm{ABC} \mathrm{pH} 8.0 \\
\text { 2. } 1 \mathrm{M} \mathrm{HA}, 4.5 \mathrm{M} \text { Gnd- } \mathrm{HCl}, 0.2 \mathrm{M} \mathrm{K}_{2} \mathrm{CO}_{3}, \mathrm{pH} 9.0\end{array}$ \\
\hline CAISU $^{17}$ & 1 & $19 \mathrm{hrs}$ & $\begin{array}{l}\text { 1. } 6 \mathrm{M} \text { Gnd- } \mathrm{HCl} \\
\text { 2. } 10 \mathrm{mM} \text { Tris- } \mathrm{HCl} \mathrm{pH} 8.5,2 \mathrm{M} \text { Gnd- } \mathrm{HCl}, 2.7 \mathrm{M} \text { Urea, } 3 \% \mathrm{ACN} \\
\text { 3. } 10 \mathrm{mM} \text { Tris- } \mathrm{HCl} \mathrm{pH} 8.5,600 \mathrm{mM} \text { Gnd- } \mathrm{HCl}, 800 \mathrm{mM} \text { Urea, } 3 \% \mathrm{ACN}\end{array}$ \\
\hline CAIS $^{18}$ & 1 & 1 day & $\begin{array}{l}\text { 1. } 8 \mathrm{M} \text { Urea, } 100 \mathrm{mM} \mathrm{ABC} \mathrm{pH} 8.0 \\
\text { 2. } 2 \mathrm{M} \text { Urea, } 100 \mathrm{mM} \mathrm{ABC} \mathrm{pH} 8.0,1000 \text { U PNGaseF }\end{array}$ \\
\hline $\mathrm{SCAD}^{20}$ & 3 & 1.7 days & $\begin{array}{l}\text { 1. } 4 \% \mathrm{SDS}, 50 \mathrm{mM} \text { Tris } \\
\text { 2. } 8 \mathrm{M} \text { Urea, } 100 \mathrm{mM} \mathrm{ABC}, \mathrm{pH} 8.0\end{array}$ \\
\hline In-Gel ${ }^{30}$ & 11 & $21 \mathrm{hrs}$ & $\begin{array}{l}\text { 1. } 25 \mathrm{mM} \mathrm{ABC}, 50 \% \text { ACN } \\
\text { 4. } 25 \mathrm{mM} / 50 \mathrm{mM} \mathrm{ABC}\end{array}$ \\
\hline In-Solution & 1 & $17 \mathrm{hrs}$ & 1. $25 \mathrm{mM} \mathrm{ABC}$ \\
\hline SPEED ${ }^{29}$ & 1 & $17 \mathrm{hrs}$ & $\begin{array}{l}\text { 1. TFA } \\
\text { 2. } 2 \mathrm{M} \text { Tris Base }\end{array}$ \\
\hline
\end{tabular}

\section{$\underline{\text { Tissue Preparation }}$}

Whole Mouse Powder (WMP) Production: Whole male C67BL/6J mouse was frozen in liquid nitrogen and fractured into approximately $1 \mathrm{~cm}^{3}$ pieces. Fur and blood were not removed from the mouse before milling. The resulting pieces were kept frozen in liquid nitrogen before milling to a fine powder under liquid nitrogen using a SPEX 6870 freezer/mill. The milled powder was kept frozen and lyophilized for 24 hours. Approximately $100 \mathrm{mg}$ aliquots of lyophilized powder were then delipidated by 4 successive extractions with $2 \mathrm{~mL} \mathrm{100 \%} \mathrm{ice-cold} \mathrm{acetone} \mathrm{and} \mathrm{briefly}$ 
dried at room temperature in a fume hood. Isolated mouse organs (heart, liver, kidney, and lung) were prepared using the same method.

$\underline{\text { Decellularization Methods }}$

\section{1-Fraction $\mathbf{A}^{4}$}

Approximately 5mg of WMP was homogenized (Bullet Blender, Model BBX24, Next Advance, Inc.) for 3 minutes on power 8 in $200 \mu \mathrm{L} / \mathrm{mg}$ of high salt buffer (50 mM Tris-HCl, $0.25 \% \mathrm{CHAPS}$, $25 \mathrm{mM}$ EDTA, $3 \mathrm{M} \mathrm{NaCl}$, pH 7.4) supplemented with $10 \mu \mathrm{L} / \mathrm{mL}$ fresh protease inhibitor (Halt ${ }^{\mathrm{TM}}$ Protease Inhibitor Cocktail, Thermo Scientific ${ }^{\mathrm{TM}}$ \#78429) with the addition of approximately 50 $1 \mathrm{~mm}$ glass beads. Homogenate was vortexed at $4^{\circ} \mathrm{C}$ for 20 minutes. Homogenized tissue was spun at $18,000 \mathrm{xg}\left(4^{\circ} \mathrm{C}\right)$ for $15 \mathrm{~min}$. The resulting supernatant was removed, and the pellet was further extracted with $1 \mathrm{ml}$ high salt buffer two times with homogenization after each buffer addition. Cellular extracts were pooled into a single soluble fraction.

\section{1-Fraction $\mathbf{B}^{29}$}

Approximately $5 \mathrm{mg}$ of WMP was placed in $200 \mu \mathrm{L} / \mathrm{mg} 10 \mathrm{mM}$ Tris- $\mathrm{HCl}(\mathrm{pH} 8.0)$ with $0.1 \%$ ethylenediamine tetraacetic acid (EDTA) and $1 \mathrm{X}$ protease inhibitors (Halt ${ }^{\mathrm{TM}}$ Protease Inhibitor Cocktail, Thermo Scientific ${ }^{\mathrm{TM}}$ \#78429) at $4^{\circ} \mathrm{C}$ for 48 h. Triton X-100 (Sigma-Aldrich \#T9284) was added to a final concentration of $3 \%$ and samples were vortexed at medium power at $4^{\circ} \mathrm{C}$ for $72 \mathrm{~h}$. The solution was changed every $24 \mathrm{~h}$ by spinning at $18,000 \mathrm{xg}\left(4^{\circ} \mathrm{C}\right)$ for $15 \mathrm{~min}$ and samples were resuspended by vortexing. The resulting supernatant from each buffer exchange was pooled into a single cellular fraction. Samples were then incubated in $50 \mathrm{mM}$ Tris-HCl pH 7.4, $10 \mathrm{mM}$ $\mathrm{MgCl}_{2}, 1 \%$ benzonase (Millipore $\# 70746$ ) at $37^{\circ} \mathrm{C}$ for $24 \mathrm{~h}$, spun at $18,000 \times \mathrm{g}\left(4^{\circ} \mathrm{C}\right.$ ) for $15 \mathrm{~min}$, 
and the supernatant was discarded. Decellularized WMP was then washed with PBS with 1X protease inhibitors for $24 \mathrm{~h}$ to remove residual reagents. All steps were conducted under continuous shaking.

\section{2-Fraction}

Approximately $5 \mathrm{mg}$ of lyophilized WMP was homogenized (Bullet Blender, Model BBX24, Next Advance, Inc.) in $200 \mu \mathrm{l} / \mathrm{mg}$ buffer 1 (150 mM NaCl, $50 \mathrm{mM}$ Tris- $\mathrm{HCl}$ (pH 7.5), 5\% glycerol, 1\% NP-40 (US Biological \#N3500), $1 \mathrm{mM} \mathrm{MgCl} 2$, $1 \mathrm{X}$ protease inhibitors (Halt ${ }^{\mathrm{TM}}$ Protease Inhibitor Cocktail, Thermo Scientific ${ }^{\mathrm{TM}}$ \#78429)) at power 8 for 3 minutes with the addition of approximately $501 \mathrm{~mm}$ glass beads and vortexed at $4^{\circ} \mathrm{C}$ for 20 minutes. Homogenized tissue was spun at $18,000 \times \mathrm{g}\left(4^{\circ} \mathrm{C}\right)$ for $15 \mathrm{~min}$ and the supernatant was collected (fraction 1$)$. After the addition of each extraction buffer, samples were resuspended for 1 minute at power 8 using the Bullet Blender. Sample was then homogenized in $200 \mu \mathrm{L} / \mathrm{mg}$ (of starting tissue dry weight) buffer 2 (50mM Tris- $\mathrm{HCl}$ ( $\mathrm{pH} 7.4$ ), 2\% CHAPS, $2 \mathrm{mM}$ EDTA, $3 \mathrm{M} \mathrm{NaCl}$, $1 \mathrm{X}$ protease inhibitors) at power 8 for 3 minutes and vortexed at $4^{\circ} \mathrm{C}$ for 15 minutes. Homogenized tissue was spun at $18,000 \times \mathrm{g}$ $\left(4{ }^{\circ} \mathrm{C}\right.$ ) for $15 \mathrm{~min}$ and the supernatant was collected (fraction 2).

\section{3-Fraction $^{19}$}

Approximately $5 \mathrm{mg}$ of lyophilized WMP was homogenized (Bullet Blender, Model BBX24, Next Advance, Inc.) in $500 \mu \mathrm{l}$ PBS containing protease inhibitors (Halt ${ }^{\mathrm{TM}}$ Protease Inhibitor Cocktail, Thermo Scientific ${ }^{\mathrm{TM}} \# 78429$ ) at power 8 for 3 minutes with the addition of approximately $501 \mathrm{~mm}$ glass beads. Homogenate was centrifuged at $16,000 \mathrm{x}$ g for 20 min at $4{ }^{\circ} \mathrm{C}$ and the supernatant was collected. After the addition of each extraction buffer, samples were resuspended for 1 minute at power 8 using the Bullet Blender. Sample was then homogenized in $500 \mu \mathrm{L}$ buffer $1(150 \mathrm{mM}$ 
$\mathrm{NaCl}, 50$ mM Tris-HCl (pH 7.5), 5\% glycerol, 1\% NP-40 (US Biological \#N3500), 1 mM MgCl 2 , 1X protease inhibitors, 1\% benzonase (Millipore \#70746)) at power 8 for 15 seconds before incubating 20 mins on ice. Homogenate was centrifuged at $16,000 \mathrm{x}$ g for $20 \mathrm{~min}$ at $4{ }^{\circ} \mathrm{C}$ and the supernatant was collected and pooled with the first wash to make fraction 1. Sample was then homogenized in $500 \mu \mathrm{L}$ buffer $2(150 \mathrm{mM} \mathrm{NaCl}, 50 \mathrm{mM}$ Tris- $\mathrm{HCl}(\mathrm{pH} 7.5), 5 \%$ glycerol, $1 \%$ NP$40,0.5 \%$ sodium deoxycholate (DOC), $0.1 \%$ SDS, $1 \mathrm{X}$ protease inhibitors, $1 \%$ benzonase) at power 8 for 15 seconds before incubating 20 mins on ice. Homogenate was centrifuged at $16,000 \mathrm{xg}$ for 20 min at $4{ }^{\circ} \mathrm{C}$ and the supernatant was collected (fraction 2). Sample was then homogenized in $500 \mu \mathrm{L}$ buffer $3(500 \mathrm{mM} \mathrm{NaCl}, 50 \mathrm{mM}$ Tris- $\mathrm{HCl}$ (pH 7.5), 5\% glycerol, 1\% NP-40, 2\% DOC, $1 \%$ SDS, $1 \mathrm{X}$ protease inhibitors, $1 \%$ benzonase) at power 8 for 15 seconds before incubating 20 mins at room temperature. Homogenate was centrifuged at $16,000 \times \mathrm{g}$ for $20 \mathrm{~min}$ at $4{ }^{\circ} \mathrm{C}$ and the supernatant was collected (fraction 3). Soluble fractions were precipitated with $80 \%$ acetone and resuspended in $8 \mathrm{M}$ urea for subsequent digestion.

\section{4-Fraction (Millipore Compartment Protein Extraction Kit, \#2145)}

$5 \mathrm{mg}$ of lyophilized WMP was homogenized (Bullet Blender, Model BBX24, Next Advance, Inc.) in $500 \mu \mathrm{l}$ of Buffer C containing protease inhibitors (Halt ${ }^{\mathrm{TM}}$ Protease Inhibitor Cocktail, Thermo Scientific ${ }^{\mathrm{TM}} \# 78429$ ) for 3 minutes on power 8 with the addition of approximately $501 \mathrm{~mm}$ glass beads. Homogenate was vortexed at power 4 for 20 min at $4{ }^{\circ} \mathrm{C}$. Homogenate was then centrifuged at $16,000 \mathrm{x}$ g for $20 \mathrm{~min}$ at $4{ }^{\circ} \mathrm{C}$ and the supernatant was collected (fraction 1) and flash frozen. After the addition of each extraction buffer, samples were resuspended for 1 minute at power 8 using the Bullet Blender. The pellet was resuspended in $400 \mu \mathrm{l}$ of Buffer $\mathrm{W}$ containing protease inhibitors and vortexed at power 4 for $20 \mathrm{~min}$ at $4^{\circ} \mathrm{C}$. Sample was centrifuged at $16,000 \mathrm{x}$ g for 20 min at $4{ }^{\circ} \mathrm{C}$ and the supernatant was discarded. The pellet was then resuspended in $150 \mu$ of Buffer 
$\mathrm{N}$ containing protease inhibitors, 1\% benzonase (Millipore \#70746) and vortexed at power 4 for $20 \mathrm{~min}$ at $4^{\circ} \mathrm{C}$. Homogenate was centrifuged at $16,000 \mathrm{x}$ g for $20 \mathrm{~min}$ at $4{ }^{\circ} \mathrm{C}$ and the supernatant was collected (fraction 2) and flash frozen. Centrifugation was repeated and the remaining supernatant was added to the $\mathrm{N}$ fraction. The pellet was resuspended in $400 \mu \mathrm{l}$ of Buffer $\mathrm{W}$ containing protease inhibitors and vortexed at power 4 for $20 \mathrm{~min}$ at $4^{\circ} \mathrm{C}$. Sample was centrifuged at $16,000 \mathrm{x}$ f for $20 \mathrm{~min}$ at $4{ }^{\circ} \mathrm{C}$ and the supernatant was discarded. The pellet was then resuspended in $100 \mu \mathrm{l}$ of Buffer $\mathrm{M}$ containing protease inhibitors and vortexed at power 4 for $20 \mathrm{~min}$ at $4^{\circ} \mathrm{C}$. Homogenate was centrifuged at $16,000 \mathrm{x}$ g for $20 \mathrm{~min}$ at $4{ }^{\circ} \mathrm{C}$ and the supernatant was collected (fraction 3) and flash frozen. The pellet was then resuspended in $200 \mu$ of Buffer CS containing protease inhibitors and vortexed at power 4 for $20 \mathrm{~min}$ at $4{ }^{\circ} \mathrm{C}$. 9 . Homogenate was centrifuged at $16,000 \mathrm{x} \mathrm{g}$ for $20 \mathrm{~min}$ at $4{ }^{\circ} \mathrm{C}$ and the supernatant was collected (fraction 4). The pellet was resuspended in $150 \mu$ of Buffer $C$ containing protease inhibitors and vortexed at power 4 for 20 $\min$ at $4{ }^{\circ} \mathrm{C}$. Homogenate was then centrifuged at $16,000 \mathrm{x}$ g for $20 \mathrm{~min}$ at $4{ }^{\circ} \mathrm{C}$ and the supernatant was pooled with fraction CS before flash-freezing the CS fraction. Additional washes were performed by resuspending the pellet in $500 \mu \mathrm{l}$ of PBS containing protease inhibitors and vortexing at power 4 for $5 \mathrm{~min}$ at $4^{\circ} \mathrm{C}$. Homogenate was centrifuged at $16,000 \mathrm{x} \mathrm{g}$ for $20 \mathrm{~min}$ at $4^{\circ} \mathrm{C}$ and the supernatant was discarded. Washes were repeated three times.

\section{Digestion and Preparation of Extracts from Decellularization for MS}

All decellularization fractions were digested using the filter aided sample preparation (FASP) protocol as previously described ${ }^{32}$ using $10 \mathrm{kDa}$ molecular weight cutoff filters (Sartorius, Vivacon \#VN01H02). Digests were performed for 16 hours in $25 \mathrm{mM} \mathrm{ABC} \mathrm{pH} 8.0$ using trypsin at a 1:100 enzyme:protein ratio at $37^{\circ} \mathrm{C}$ in an oven. Digested samples were desalted using Pierce ${ }^{\mathrm{TM}} \mathrm{C} 18$ Spin Tips (Thermo Scientific \#84850) according to the manufacturer's protocol. 


\section{$\underline{\text { Single-Shot Methods }}$}

\section{Surfactant and Chaotropic Agent Assisted Sequential Extraction/On-pellet Digestion $(\text { SCAD })^{21}$}

Approximately $5 \mathrm{mg}$ of WMP was solubilized in $300 \mu \mathrm{L}$ of buffer (4\% SDS, $50 \mathrm{mM}$ Tris buffer) and incubated at $95{ }^{\circ} \mathrm{C}$ for $10 \mathrm{~min}$. After allowing the solution to return to room temperature, protein extract was reduced with $10 \mathrm{mM}$ dithiothreitol (DTT) for $30 \mathrm{~min}$ at room temperature and alkylated with $50 \mathrm{mM}$ iodoacetamide (IAM) for an additional $15 \mathrm{~min}$ in the dark. The reaction was then quenched with an additional $2 \mathrm{mM}$ DTT. SDS was removed by two rounds of precipitation. For the first precipitation, cold acetone $\left(-20^{\circ} \mathrm{C}\right)$ was added to a final concentration of $80 \%(\mathrm{v} / \mathrm{v})$, and the protein was precipitated overnight at $-20{ }^{\circ} \mathrm{C}$. For the second round, $80 \%$ acetone/water (v/v) was added, followed by incubation at $-20^{\circ} \mathrm{C}$ for $2 \mathrm{~h}$. The samples were centrifuged at 18,000 $\times \mathrm{g}$ for $15 \mathrm{~min}$, and the pellet was briefly air-dried in a fume hood. Pellet was dissolved in $125 \mathrm{uL}$ of 8M urea and on-pellet digestion was performed with Lys-C (1:100, Wako \#121-05063) for $4 \mathrm{~h}$ at $37{ }^{\circ} \mathrm{C}$. Samples were diluted with $875 \mathrm{uL}$ of $50 \mathrm{mM}$ Tris buffer along with Trypsin $(1: 100$, Promega \#V511C) for overnight digestion. The reaction was quenched with 1\% FA. Digested samples were desalted using Pierce ${ }^{\mathrm{TM}} \mathrm{C} 18$ Spin Tips (Thermo Scientific \#84850) according to the manufacturer's protocol.

\section{In-Gel Digest ${ }^{31}$}

Approximately $1 \mathrm{mg}$ of WMP was suspended in SDS-PAGE loading buffer (5\% SDS, $250 \mathrm{mM}$ Tris- $\mathrm{HCl} \mathrm{pH} \mathrm{7.0,50 \%} \mathrm{glycerol)} \mathrm{and} \mathrm{heated} \mathrm{to} 95^{\circ} \mathrm{C}$ for 5 minutes. The protein homogenate was then loaded onto a 3-8\% TAE gel and run $1 \mathrm{~cm}$ into the gel. The gel was stained with Brilliant Blue R (Sigma-Aldrich \#B7920) and the entire protein-containing band was excised. In-gel 
digestion was performed as previously described. ${ }^{31}$ Digested samples were desalted using Pierce ${ }^{\mathrm{TM}}$ C18 Spin Tips (Thermo Scientific \#84850) according to the manufacturer's protocol.

\section{In-Solution Digest}

Approximately $1 \mathrm{mg}$ of WMP was suspended in 20uL of 50mM ABC, 0.2\% ProteaseMax. Sample was vortexed for 60 minutes. Sample was diluted with 100uL 50mM ABC. DTT was added to the sample to reach a final concentration of $10 \mathrm{mM}$ and incubated at $37^{\circ} \mathrm{C}$ for 15 minutes. Sample was removed from heat and allowed to cool for 5 minutes to condense and then spun down to collect condensate. IAM was added to sample at 2.5 molar excess DTT and incubated in the dark at room temperature for 30 minutes. Alkylation was quenched with $10 \%$ excess DTT. Digestion was performed using 3 ug Trypsin in $0.03 \%$ ProteaseMax overnight at $37^{\circ} \mathrm{C}$. Samples were then acidified to $0.1 \%$ FA to stop digestion. Digested samples were desalted using Pierce ${ }^{\mathrm{TM}} \mathrm{C} 18$ Spin Tips (Thermo Scientific \#84850) according to the manufacturer's protocol.

\section{Sample Preparation by Easy Extraction and Digestion (SPEED) ${ }^{30}$}

Approximately $5 \mathrm{mg}$ of WMP was suspended in $100 \mu \mathrm{L}$ trifluoroacetic acid (TFA) and incubated at room temperature for $10 \mathrm{~min}$. Samples were further irradiated for $10 \mathrm{~s}$ at $800 \mathrm{~W}$ using a microwave oven. Samples were neutralized with 2M TrisBase using 10x volume of TFA before adding DTT to $10 \mathrm{mM}$ and reducing for 30 minutes at $37^{\circ} \mathrm{C}$. Iodoacetamide was added to a $2.5 \mathrm{x}$ molar excess over DTT and samples were incubated in the dark for 15 minutes. Digestion was carried out for $20 \mathrm{hrs}$ at $37^{\circ} \mathrm{C}$ using trypsin at an enzyme:protein ratio of 1:50. Digested samples

were desalted using Pierce ${ }^{\mathrm{TM}}$ C18 Spin Tips (Thermo Scientific \#84850) according to the manufacturer's protocol. 


\section{$\underline{\text { ECM Extraction Methods }}$}

\section{Hydroxylamine Chemical Digest (Gnd-HCl/HA) ${ }^{4}$}

ECM-enriched pellets were homogenized in $6 \mathrm{M}$ guanidine hydrochloride (Gnd-HCl), 100mM ammonium bicarbonate $(\mathrm{ABC})$ at power 8 for 1 minute (Bullet Blender, Model BBX24, Next Advance, Inc.) and vortexed (power 5) at room temperature overnight. Homogenate was spun at $18,000 \times \mathrm{g}\left(4^{\circ} \mathrm{C}\right)$ for $15 \mathrm{~min}$ and the supernatant was collected as the $\mathrm{Gnd}-\mathrm{HCl}$ fraction. Remaining pellets were reduced and alkylated by incubating in $10 \mathrm{mM}$ DTT, $100 \mathrm{mM} \mathrm{ABC} \mathrm{pH} 8.0$ for 30 minutes at $37^{\circ} \mathrm{C}$ before adding $2.5 \mathrm{x}$ molar excess of IAM (over DTT) and incubating in the dark for 15 minutes. Samples were spun at $18,000 \times \mathrm{g}\left(4^{\circ} \mathrm{C}\right)$ for $15 \mathrm{~min}$ and the supernatant was discarded. Pellets were then treated with freshly prepared hydroxylamine (HA) buffer (1 M $\mathrm{NH}_{2} \mathrm{OH}-\mathrm{HCl}, 4.5 \mathrm{M}$ Gnd- $\mathrm{HCl}, 0.2 \mathrm{M} \mathrm{K}_{2} \mathrm{CO}_{3}, \mathrm{pH}$ adjusted to 9.0 with $\mathrm{NaOH}$ ) at $200 \mu \mathrm{L} / \mathrm{mg}$ of the starting tissue dry weight. Each tube was placed under a stream of nitrogen gas and sealed before being homogenized at power 8 for 1 minute and incubated at $45^{\circ} \mathrm{C}$ with shaking $(1000$ rpm) for $4 \mathrm{~h}$. Following incubation, the samples were spun for $15 \mathrm{~min}$ at $18000 \mathrm{x}$, and the supernatant was removed and stored as the hydroxylamine (HA) fraction at $-80^{\circ} \mathrm{C}$ until further proteolytic digestion with trypsin. All Gnd-HCl and $\mathrm{HA}$ fractions were subsequently subjected to enzymatic digestion with trypsin using a filter aided sample preparation (FASP) approach ${ }^{32}$ and desalted using Pierce ${ }^{\mathrm{TM}}$ C18 Spin Tips (Thermo Scientific \#84850) according to the manufacturer's protocol.

\section{Chaotrope-assisted In-solution Digest with Ultrasonication (CAISU) ${ }^{19}$}

Digestion of ECM-enriched pellets was performed in two steps. Samples were resuspended in 6M guanidinium hydrochloride (Gnd-HCl). Samples were reduced with 10mM DTT for 30 minutes at 
room temperature and alkylated with 50mM IAM for 15 minutes in the dark and the reaction was quenched with additional DTT. The first digestion was done at $37^{\circ} \mathrm{C}$ for $2 \mathrm{hrs}$ with LysC (1:50 enzyme to protein ratio) in $10 \mathrm{mM}$ Tris- $\mathrm{HCl}(\mathrm{pH} 8.5)$ containing $2 \mathrm{M}$ Gnd-HCl, $2.7 \mathrm{M}$ urea, and $3 \%$ acetonitrile. Prior to the two-hour incubation, samples were sonicated for 15 minutes $\left(37^{\circ} \mathrm{C}\right)$ using a Bioruptor plus ultrasonicator (Diagenode). The second digestion step was done using fresh LysC (1:50 enzyme to protein ratio) and trypsin (1:20 enzyme to protein ratio) in $600 \mathrm{mM}$ Gnd$\mathrm{HCl}, 800 \mathrm{mM}$ urea, and $3 \%$ acetonitrile at $37^{\circ} \mathrm{C}$ overnight. An additional sonication step was also performed prior to the overnight digest. Digested samples were desalted using Pierce ${ }^{\mathrm{TM}} \mathrm{C} 18$ Spin Tips (Thermo Scientific \#84850) according to the manufacturer's protocol.

\section{Chaotrope-assisted In-solution Digest (CAIS) ${ }^{20}$}

ECM-enriched pellets were resuspended in $50 \mu \mathrm{L}$ of $8 \mathrm{M}$ urea, $100 \mathrm{mM} \mathrm{ABC}, 10 \mathrm{mM}$ DTT and incubated with continuous agitation at $1,400 \mathrm{rpm}$ for $2 \mathrm{hrs}$ at $37^{\circ} \mathrm{C}$. Samples were cooled to RT and $500 \mathrm{mM}$ IAM in water was added to a final concentration of $25 \mathrm{mM}$. Samples were then incubated in the dark for 30 min at RT. Sample buffer was diluted to $2 \mathrm{M}$ urea with $100 \mathrm{mM} \mathrm{ABC}$ pH 8.0 and 1000U PNGaseF was added before incubating with continuous agitation at 1,400 rpm for $2 \mathrm{hr}$ at $37^{\circ} \mathrm{C}$. Lys-C $(1 \mu \mathrm{g})$ was added and samples were incubated with continuous agitation at 1,400 rpm for $2 \mathrm{hr}$ at $37^{\circ} \mathrm{C}$. Trypsin $(3 \mu \mathrm{g})$ was added and samples were incubated with continuous agitation at $1,400 \mathrm{rpm} \mathrm{O} / \mathrm{N}$ at $37^{\circ} \mathrm{C}$. A second aliquot of trypsin $(1.5 \mu \mathrm{g})$ was added and samples were incubated with continuous agitation at 1,400 rpm for an additional $2 \mathrm{hrs}$ at 37 ${ }^{\circ} \mathrm{C}$. Trypsin was then inactivated by acidifying the sample with $10 \%$ formic acid. Acidified samples were centrifuged at $16,000 \mathrm{x}$ g for $5 \mathrm{~min}$ at RT and the supernatant was collected. Digested samples were desalted using Pierce ${ }^{\mathrm{TM}} \mathrm{C} 18$ Spin Tips (Thermo Scientific \#84850) according to the manufacturer's protocol. 
SCAD $^{21}$

Protocol was performed as described above using WMP pellets decellularized using the 1-fraction A method rather than WMP.

\section{Sample Preparation of Isolated Organs for Instrument Comparison}

Samples of isolated heart, kidney, and liver from C57BL/6J mice were dissected and flash frozen by the Jackson Lab. Each organ was cryo-milled into a fine, homogenous powder using a mortar and pestle under liquid nitrogen and lyophilized for future processing. Organ samples from 3 individual mice were pooled and mixed thoroughly before weighing. Samples were decellularized using the 1-fraction A method, as described above, followed by the Gnd-HCl/HA, CAISU, CAIS, and SCAD ECM extraction methods. All ECM extraction methods were performed as previously described for WMP comparisons.

ECM Extraction Method Optimization

\section{Gnd-HCl/HA Digest Optimization}

For antioxidant testing, hydroxylamine digest was performed as described above with the addition of $50 \mathrm{mM}$ methionine, $100 \mu \mathrm{M} / 500 \mu \mathrm{M}$ caffeic acid, $100 \mu \mathrm{M} / 500 \mu \mathrm{M}$ gallic acid, or $2 \mathrm{mM}$ ascorbic acid (vitamin C) to the hydroxylamine digest buffer immediately before pellet treatment. Acid pretreatment testing was performed by incubating the insoluble pellet in $0.2 \%$ formic acid (FA) for 10 minutes before spinning at $18,000 \times \mathrm{g}\left(4^{\circ} \mathrm{C}\right)$ for $15 \mathrm{~min}$ and discarding the supernatant. For each condition, HA buffer at the stated hydroxylamine concentration was $\mathrm{pH}$-adjusted prior to pellet treatment and the digest was carried out for the stated time under vortexing. 


\section{PNGase F Digestion}

PNGase F digestion was performed on a $10 \mathrm{kDa}$ cutoff filter (Sartorius \#VN01H02) prior to FASP digestion. Filters were first equilibrated with successive washes of $0.1 \%$ FA followed by $8 \mathrm{M}$ urea, $100 \mathrm{mM}$ ABC $\mathrm{pH}$ 8.0. All washes were spun through at $14,000 \mathrm{x} \mathrm{g}$ for 15 minutes. Samples were then loaded onto filters and spun at $14,000 \mathrm{x}$ g for 20 minutes before washing with $200 \mu \mathrm{L}$ of $8 \mathrm{M}$ urea, $100 \mathrm{mM}$ ABC. Filters were then washed with 3 aliquots of $100 \mu \mathrm{L} 50 \mathrm{mM}$ ABC pH 8.0 before adding 1000U PNGase F (NEB \#P0704) in 2M urea, $100 \mathrm{mM} \mathrm{ABC} \mathrm{pH} 8.0$ directly to the top of the filter membrane. PNGase F digestion was allowed to proceed for 2 hours at $37^{\circ} \mathrm{C}$ before spinning at $14,000 \mathrm{x}$ g for 15 minutes to remove digest volume, retaining deglycosylated protein on the membrane. Filters were then washed with $100 \mu \mathrm{L} 50 \mathrm{mM}$ ABC pH 8.0 to remove residual buffer. Trypsin digest was performed in $20 \mathrm{mM} \mathrm{ABC} \mathrm{pH} 8.0$ with $0.02 \%$ ProteaseMax (Promega \#V207A) for 16 hours using a 1:100 enzyme:protein ratio. Samples were eluted and acidified in $150 \mu \mathrm{L}$ 0.2\% FA. Digested samples were desalted using Pierce ${ }^{\mathrm{TM}}$ C18 Spin Tips (Thermo Scientific \#84850) according to the manufacturer's protocol.

\section{PNGase F Digestion with GAG Removal}

Removal of N-linked glycans and glycosaminoglycans (GAGs) using PNGaseF (NEB \#P0704), heparinase II (NEB \#P0736), and chondroitinase ABC (Sigma-Aldrich \#C3667) was performed across multiple digestion steps. Filter equilibration, sample loading, and pre-digest washes were performed as described above for the PNGase F digestion. After performing three washes with 50 mM ABC, 1000U PNGase F and 0.5U heparinase II in $100 \mu \mathrm{L} 100 \mathrm{mM} \mathrm{NaCl}, 20 \mathrm{mM}$ Tris-HCl, $1.5 \mathrm{mM} \mathrm{CaCl} 2$ was added directly to each filter membrane. Digests were carried out for 16 hours at $37^{\circ} \mathrm{C}$ before spinning for 15 minutes at $14,000 \times \mathrm{g}$ to remove digest volume, retaining 
deglycosylated protein on the membrane. The filter was then washed with $100 \mu \mathrm{L} 50 \mathrm{mM}$ Tris$\mathrm{HCl}$ pH 7.5 before adding $100 \mu \mathrm{L}$ of $50 \mathrm{mM}$ Tris- $\mathrm{HCl} \mathrm{pH} 7.5,60 \mathrm{mM}$ sodium acetate, $0.02 \% \mathrm{BSA}$ containing $0.5 \mathrm{U}$ chondroitinase $\mathrm{ABC}$. Chondroitinase $\mathrm{ABC}$ digestion was performed for 8 hours at $37^{\circ} \mathrm{C}$ before spinning filters for 15 minutes at $14,000 \mathrm{x}$ g to remove digest volume. Filters were then washed with $100 \mu \mathrm{L} 50 \mathrm{mM}$ ABC pH 8.0. Trypsin digest was performed by adding trypsin at a 1:100 enzyme:protein ratio in $100 \mu \mathrm{L} 25 \mathrm{mM}$ ABC pH 8.0, 0.02\% ProteaseMax (Promega \#V2071) and allowing the digest to proceed for 16 hours at $37^{\circ} \mathrm{C}$. Samples were eluted and acidified in $150 \mu \mathrm{L} 0.2 \%$ FA. Digested samples were desalted using Pierce ${ }^{\mathrm{TM}} \mathrm{C} 18$ Spin Tips (Thermo Scientific \#84850) according to the manufacturer's protocol.

$\underline{\text { Data Acquisition and Processing }}$

\section{Sample Quantification and Normalization}

Samples from all methods were quantified after trypsin digest using a Micro BCA protein assay kit (Thermo Fisher Scientific \#23235) according to the manufacturer's protocol. Aliquots containing $10 \mu \mathrm{g}$ of protein from each digest were loaded onto PierceTM C18 Spin Tips (Thermo Scientific \#84850) and desalted using the manufacturer's protocol. Each sample was dried to approximately $2 \mu \mathrm{L}$ under vacuum and was brought up in $16 \mu \mathrm{L} 0.1 \%$ FA.

\section{MS/MS Acquisition}

Global proteomics for all comparative method testing was carried out ( $\mathrm{n}=3$ per group) on an LTQ Orbitrap Velos mass spectrometer (Thermo Fisher Scientific) coupled to an Eksigent nanoLC-2D system through a nanoelectrospray LC - MS interface. Eight $\mu \mathrm{L}$ of each sample was injected into a $20 \mu \mathrm{L}$ loop using the autosampler. The analytical column was then switched on-line at $600 \mathrm{nl} / \mathrm{min}$ 
over an in house-made $100 \mu \mathrm{m}$ i.d. $\times 150 \mathrm{~mm}$ fused silica capillary packed with $2.7 \mu \mathrm{m}$ CORTECS C18 resin (Waters; Milford, MA). After $10 \mathrm{~min}$ of sample loading at $600 \mathrm{~nL} / \mathrm{min}$, each sample was separated on a 120 -min gradient consisting of a linear gradient from $2-8 \%$ ACN with $0.1 \%$ formic acid (FA) at a flow rate of $600 \mathrm{~nL} / \mathrm{min}$ from 3 minutes to 20 minutes, followed by a linear gradient from $8-22 \% \mathrm{ACN}$ with $0.1 \% \mathrm{FA}$ at a flow rate of $350 \mathrm{~nL} / \mathrm{min}$ from 20 minutes to 90 minutes. Gradient elution was followed by a linear increase to $60 \% \mathrm{ACN}$ at $350 \mathrm{~nL} / \mathrm{min}$ from 90 minutes to 98 minutes and further to $90 \% \mathrm{ACN}$ from 98 minutes to 104 minutes to remove remaining peptides. The column was then re-equilibrated with $2 \% \mathrm{ACN}$ in $0.1 \% \mathrm{FA}$ at $350 \mathrm{~nL} / \mathrm{min}$ from minutes 104 to 120 . LC mobile phase solvents consisted of $0.1 \%$ formic acid in water (Buffer A) and $0.1 \%$ formic acid in acetonitrile (Buffer B, Optima ${ }^{\mathrm{TM}}$ LC/MS, Fisher Scientific, Pittsburgh, PA). Data acquisition was performed using the instrument supplied Xcalibur ${ }^{\mathrm{TM}}$ (version 4.1) software. The mass spectrometer was operated in the positive ion mode. Each survey scan of $\mathrm{m} / \mathrm{z}$ 300-2,000 was followed by collision-induced dissociation (CID) MS/MS of the 20 most intense precursor ions with an isolation width of $2.5 \mathrm{~m} / \mathrm{z}$. Dynamic exclusion was performed after fragmenting a precursor 2 times within $15 \mathrm{sec}$ for a duration of $30 \mathrm{sec}$. Singly charged ions were excluded from CID selection. Normalized collision energies of $35 \mathrm{eV}$ were employed using helium as the collision gas.

timsTOF Pro data acquisition for isolated organs was carried out ( $n=3$ per group) using a Bruker NanoElute LC system through a nanoelectrospray LC-MS interface. One $\mu \mathrm{L}$ of each sample was injected into a $20 \mu \mathrm{L}$ loop using the autosampler. The analytical column was then switched on-line at $600 \mathrm{nl} / \mathrm{min}$ over a $15 \mathrm{~cm}$ NanoElute column (Bruker Daltonics) using ReproSil $1.9 \mu \mathrm{m}$ C18 resin (Dr. Maisch GmbH, Germany). After 4 min of sample loading at 800.0 bar, each sample was separated on a 120-min gradient. For cellular and Gnd-HCl fractions, the LC method consisting of 
a linear gradient from $2-24 \% \mathrm{ACN}$ with $0.1 \%$ formic acid (FA) at a flow rate of $500 \mathrm{~nL} / \mathrm{min}$ from 2 minutes to 112 minutes, followed by a linear increase to $95 \% \mathrm{ACN}$ at $500 \mathrm{~nL} / \mathrm{min}$ from 112 minutes to 115 minutes. Column washing at 95\% ACN was performed from minutes 115 to 120 . For HA fractions, the same LC method was followed but with a linear gradient from $2-20 \%$ ACN from 2 minutes to 112 minutes. LC mobile phase solvents consisted of $0.1 \%$ formic acid in water (Buffer A) and $0.1 \%$ formic acid in acetonitrile (Buffer B, Optima ${ }^{\mathrm{TM}} \mathrm{LC} / \mathrm{MS}$, Fisher Scientific, Pittsburgh, PA). Data acquisition was performed using the manufacturer-supplied otofControl (version 6.0) software with the instrument default data-dependent acquisition (DDA) parallel accumulation-serial fragmentation (PASEF) method with a cycle time of $1.1 \mathrm{~s}$. The mass spectrometer was operated in the positive ion mode. In brief, each survey scan of $\mathrm{m} / \mathrm{z} 100-1,700$ was followed by 10 PASEF MS/MS scans employing collision-induced dissociation (CID). Active exclusion was performed with an intensity threshold of $2500 \mathrm{cts} / \mathrm{s}$ and releasing after $0.2 \mathrm{~min}$, reconsidering precursors if the current intensity is 4-fold greater than the previous intensity.

\section{Data Processing}

All Orbitrap-acquired raw MS files were converted to .mgf format using Proteome Discoverer (Thermo Fisher Scientific) using the default parameters. Converted files were then searched using an in-house Mascot ${ }^{\mathrm{TM}}$ server (Version 2.5, Matrix Science). For Orbitrap acquired data mass tolerances were $\pm 15 \mathrm{ppm}$ for MS peaks, and $\pm 0.6 \mathrm{Da}$ for MS/MS fragment ions. Protein probability thresholds were set at $99.9 \%$ with a minimum of 2 peptides and peptide thresholds were set at 95\% using local false discovery rate (LFDR) scoring implemented in Scaffold (version 4.9.0, Proteome Software Inc.), resulting in a protein FDR of $0.0 \%$ and a peptide FDR of $0.5 \%$. For timsTOF Pro acquired raw files, searching was performed using Peaks Studio (Version 10.5, Bioinformatics Solutions Inc.). Data refinement was performed by correcting precursor mass only, 
associating features with chimera scans, and filtering features for charge between 2 and 8 . PeaksDB search was performed using $\pm 15 \mathrm{ppm}$ for MS peaks, and \pm 0.1 Da for MS/MS fragment ions. Data was filtered to $1 \%$ FDR at the peptide level and protein probability threshold was set to $\mathrm{P} \leq 0.01$ using PEAKS.

For all searches, data was searched against SwissProt (17,029 sequences) restricted to $M u s$ musculus using version 1.1 of the CRAPome for common contaminants. ${ }^{33}$ Trypsin specific cleavage was used in searches for cellular and enzyme-extracted ECM fractions, while HA/Trypsin specificity was used for hydroxylamine digested fractions, both allowing for 2 missed cleavages. HA/Trypsin specificity was defined as cleaving $\mathrm{C}$-terminal of $\mathrm{K}$ and $\mathrm{R}$ but not before $\mathrm{P}$, as well as C-terminal of $\mathrm{N}$ but not before $\mathrm{C}, \mathrm{F}, \mathrm{H}, \mathrm{I}, \mathrm{M}, \mathrm{N}, \mathrm{Q}, \mathrm{S}, \mathrm{W}$, or $\mathrm{Y}$ based on previous HA cleavage data. ${ }^{4}$ Fixed modifications were set as carbamidomethyl (C). Variable modifications were set as oxidation (M), oxidation (P) (hydroxyproline), Gln->pyro-Glu (N-term), deamidated (NQ), and acetyl (Protein N-term).

To identify additional experimentally-induced modifications, Mascot searches were performed as described above with additional variable modifications (Search 1: Oxidation (D), Oxidation (HW), Oxidation (K), Oxidation (R); Search 2: Carbamyl (K), Carbamyl (R), Carbamyl (N-term)).

\section{Data Analysis}

Protein Classification: Core matrisome annotations, including collagens, proteoglycans, and ECM glycoproteins, were mapped using the mouse matrisome database (MatrisomeDB, http://matrisomeproject.mit.edu/). ${ }^{28}$ Cellular proteins are defined as all proteins not annotated within the MarisomeDB as core matrisome or matrisome-associated. 
Normalization to Equal Run Time: To compare methods generating different numbers of fractions, PSMs were normalized to total run time by dividing the sum of PSMs in all fractions of a single method by the number of fractions analyzed.

Weighted Average Sequence Coverage: Sequence coverage was determined using an in-house $\operatorname{Mascot}^{\mathrm{TM}}$ server (Version 2.5, Matrix Science) and Scaffold (version 4.9.0, Proteome Software Inc.). For each round of sample runs, fractional abundance of a given protein was calculated by dividing peptide spectral matches for that protein by the total number of peptide spectral matches for proteins in that category (i.e. collagens) across all compared samples. The sequence coverage for an individual protein was then multiplied by the fractional abundance. The weighted sequence coverage for proteins in that category were summed to provide the weighted average sequence coverage for that category.

Statistical Significance: P-values were calculated using a two-tailed equal variance student's t-test. P-values in text are reported as the least significant relevant comparison. In figures, “*” denotes $\mathrm{p}<0.05$, “**” denotes $\mathrm{p}<0.01$, and “***” denotes $\mathrm{p}<0.001$.

Overall Method Scoring: Overall method scores were determined by scoring each method based on a variety of relevant criteria and calculating a weighted average of these scores. Each category is scored from 1 to 10 , with 1 representing the lowest possible score and 10 representing the highest. For categories "Time" and "ECM in Cellular Fraction", higher scores are given to methods with lower actual values. Decellularization was scored based on time (10\%), ease (10\%), MS compatibility of buffers (10\%), total protein identification (30\%), precision (20\%), and ECM in the cellular fraction (20\%). For comparison to decellularization methods, single-shot methods were compared on all metrics except ECM in the cell fraction, dividing the weight for 
this metric evenly among the other categories. ECM extraction methods were scored based on time (10\%), ease (10\%), collagen identification (30\%), glycoprotein and proteoglycan identification (30\%), and precision (20\%). Identical scoring and weighting were applied to single-shot methods for comparison to ECM extraction methods.

\section{$\underline{\text { Results }}$}

\section{Evaluation of Cellular Protein Extraction Methods for ECM Enrichment}

To compare the various decellularization methods, we evaluated extraction of cellular proteins and preservation of ECM proteins for subsequent ECM analysis. Whole mouse powder (WMP) was chosen for method development as it is a complex mixture that includes a representative sampling of all tissues and covers matrix components across a wide dynamic range. The single-shot methods were also compared for extraction of cellular proteins (Figure 1A). For decellularization methods with multiple fractions, each fraction was analyzed separately, and data is presented as both fraction sums and per-fraction averages. When normalized to equal MS run time, the 1-fraction A method generates more cellular peptide spectral matches (PSMs) than other methods, with 15\% more PSMs than the next-highest 4-fraction method $(\mathrm{p}=0.014)$, while in-solution digest yields the least (Figure 1B). No significant differences in cellular spectral matches were observed between 2-fraction, 3-fraction, and 4-fraction extractions when comparing per-fraction averages. Of note, the 4-fraction method displayed significantly higher variability than other methods, attributed to one outlier sample. All tested ECM extraction protocols were repeated in triplicate with new starting material and the results were consistent based on performance comparisons against the 1fraction A method (Supporting Information Figure 1). Therefore, the observed variability is likely attributed to the method rather than an error in sample preparation. The 1-fraction B method 
resulted in significantly fewer cellular PSMs than any other decellularization method $(\mathrm{p}=0.0052)$.

With increased decellularization fractions, there is roughly a linear increase in total cellular PSMs

(Figure 1B). In comparison of single-shot methods, in-gel and SPEED digestion result in the most cellular PSMs ( $\mathrm{p}=0.0043$ and 0.0027 respectively), although all single-shot extractions generate fewer cellular PSMs than the 1-fraction, 2-fraction, and 4-fraction decellularization methods when normalized to equal run time (Figure 1B).

As stated, decellularization methods prior to ECM analysis should efficiently extract highabundance cellular proteins while minimizing ECM extraction for subsequent analysis. Therefore, any ECM proteins removed by decellularization would be considered adverse for accuracy of subsequent ECM protein analysis. The 1-fraction B method results in the lowest number of core matrisome PSMs identified in the cellular fraction $(\mathrm{p}=0.026)$ (Figure 1C). This finding, in addition to the low number of cellular PSMs identified using the 1-fraction B method, indicates this method provides the mildest decellularization conditions of all the tested methods. In contrast, the 1fraction A and 4-fraction methods extract the largest amount of core matrisome PSMs per fraction in their cellular fractions, although increased variability in the 4-fraction method prevents significance from being reached (Figure 1C). Significantly more core matrisome PSMs were identified in the second fraction of the 4-fraction decellularization method than in any individual cellular fraction from another method $\left(136 \%\right.$ greater, $\left.\mathrm{p}=8.5 \times 10^{-5}\right)$, confirming that this method extracts the largest amount of ECM proteins during decellularization. For all decellularization methods, greater than $75 \%$ of PSMs of proteins classified as matrisome-associated ${ }^{28}$ were $^{2}$ identified in the cellular fractions (Supporting Information Figure 2). The solubility of matrisomeassociated proteins in all tested decellularization buffers supports the decision to focus on the core matrisome when evaluating ECM extraction and analysis. 


\section{Evaluation of Resulting ECM Fractions Following Various Decellularization Approaches}

To facilitate comparison of the remaining ECM pellets from each decellularization method, the protein composition was assessed via a single approach (the guanidine hydrochloride extraction followed by hydroxylamine digestion (Gnd-HCl/HA) method) for ECM analysis (Figure 1A, Table 1). The most effective decellularization method should result in high sequence coverage for core ECM components such as collagen, proteoglycans, and glycoproteins within the ECM fractions. The 4-fraction decellularization method resulted in 45\% higher collagen PSMs within the subsequent ECM fractions than other methods $(p=0.0029)$ (Supporting Information Figure 3A). However, it did not produce significantly more unique collagen peptides than other methods (Figure 1D). No significant difference in unique collagen peptides within the ECM fractions was identified between any tested methods (Figure 1D). The 1 -fraction A (14\% greater, $\mathrm{p}=0.011)$ and 2-fraction $(9 \%$ greater, $\mathrm{p}=0.022)$ methods provide more unique peptides for proteoglycans and ECM glycoproteins, while the 1-fraction B and 4-fraction methods generate $12 \%$ fewer unique peptides than other methods (Figure 1E). No significant differences in ECM glycoprotein and proteoglycan PSMs were identified between any tested methods (Supporting Information Figure 3B). 

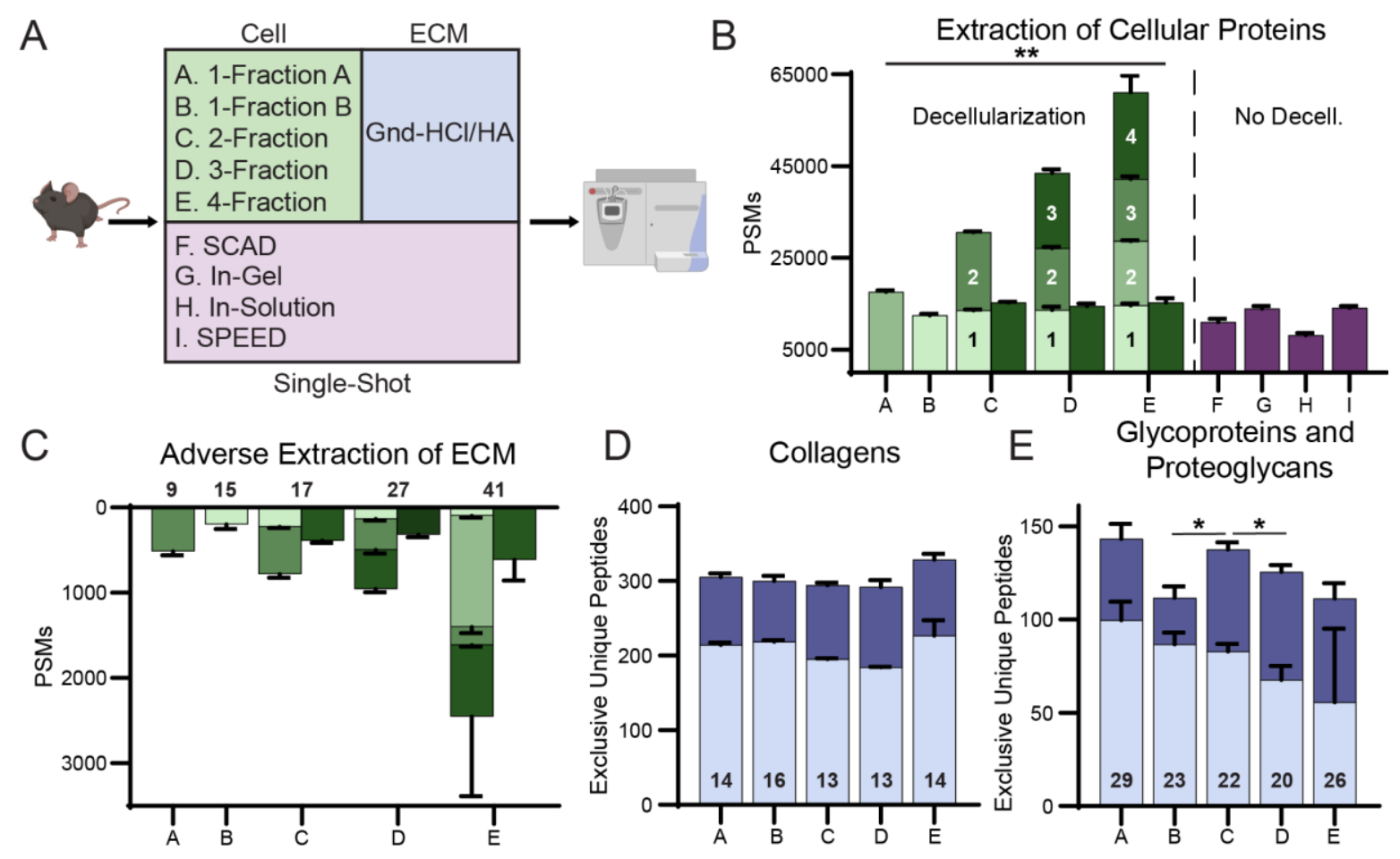

Figure 1. Effects of soluble protein extraction methods on extracellular matrix identification. A) Workflow of decellularization and single-shot methods used. All decellularization methods were followed by a single ECM analysis method (Gnd-HCl/HA extraction). B) Total cellular PSMs identified by each decellularization (green) and single-shot (purple) method. Multi-step methods are shown as both a sum of all fractions and the average PSMs per fraction. C) Total ECM PSMs lost during decellularization. Multi-step methods are shown as both a sum of all fractions and the average PSMs per fraction. Numbers above bars indicate the number of core matrisome proteins identified with a minimum of 2 peptides. D) Total exclusive unique collagen peptides identified in the subsequent $\mathrm{Gnd}-\mathrm{HCl} / \mathrm{HA} \mathrm{ECM}$ extraction following each decellularization method. Light blue indicates $\mathrm{Gnd}-\mathrm{HCl}$ fraction, dark blue indicates new unique peptides from HA fraction. Numbers on bars indicate the number of distinct collagen chains identified with a minimum of 2 peptides. E) Total exclusive unique peptides for proteoglycans and glycoproteins identified in the subsequent ECM extraction following each decellularization method. Numbers on bars indicate the number of ECM glycoproteins and proteoglycans identified with a minimum of 2 peptides. All bar plots present group averages with standard deviation (SD).

\section{Evaluation of ECM Extraction and Digestion Methods}

To assess ECM extraction methods on uniform starting material, a single decellularization method (1-fraction A) was performed on all samples prior to the four ECM extraction methods under evaluation (Figure 2A). Comparing all methods, the Gnd-HCl/HA method was found to result in the greatest number of identified collagen proteins, unique peptides, and PSMs even when 
normalized to equal run time, providing $37 \%$ more unique collagen peptides than the next best method: SCAD post-decellularization $(\mathrm{p}=0.0002)$ (Figure 2B,C). Additionally, Gnd-HCl/HA $(p=0.0008)$ and SCAD post-decellularization $(p=0.045)$ extractions generate significantly more sequence coverage of collagen proteins than the CAIS extraction method (Supporting Information Figure 4A). The CAISU and CAIS extractions resulted in similar numbers of unique collagen peptide identifications (Figure 2C). However, the CAISU method produced the lowest number of spectral matches for collagen peptides of the tested post-decellularization ECM extractions $(p=0.0002)$ and also produced fewer collagen PSMs than the SCAD $(p=0.0032)$ and SPEED $(p=0.0029)$ single-shot methods despite their lack of cellular protein removal (Figure 2B). Singleshot protocols SCAD and SPEED performed similarly in all assessed metrics for collagen extraction (Figure 2B,C). Decellularization prior to the SCAD protocol offered significant improvement in identification of collagen spectral matches $(p=0.0039)$ and unique collagen peptides $(p=0.0024)$ (Figure $2 B, C)$. Single-shot in-solution digestion resulted in the lowest number of collagen PSMs and unique peptides (Figure 2B). When comparing individual fractions, the HA fraction from the Gnd-HCl/HA extraction yielded the most collagen PSMs $(p=0.0042)$ (Figure 2B).

Identification of glycoproteins and proteoglycans was also assessed to determine the best method for comprehensive core ECM analysis. The two-fraction $\mathrm{Gnd}-\mathrm{HCl} / \mathrm{HA}$ method resulted in the identification of more glycoproteins and proteoglycans (21) than any other method with 74\% more unique peptides than the next best method, CAISU $(\mathrm{p}=0.0001)$ (Figure 2E). Additionally, the Gnd$\mathrm{HCl} / \mathrm{HA}$ extraction generated a $25 \%$ increase in glycoprotein and proteoglycan sequence coverage $(p=0.024)$ (Supporting Information Figure 4B) and 10\% more PSMs $(p=0.011)$ than the next best method, even when normalized to equal run time (Figure 2D). SCAD extraction post- 
decellularization, on the other hand, produced significantly fewer unique peptides for glycoproteins and proteoglycans than both the $\mathrm{Gnd}-\mathrm{HCl} / \mathrm{HA}(\mathrm{p}=0.00015)$ and CAISU $(\mathrm{p}=0.0051)$ methods (Figure 2E). SPEED extraction produced 14.5\% higher glycoprotein and proteoglycan PSMs than other single-shot methods (Figure 2D), although this method identified significantly fewer unique peptides in these categories than either the Gnd-HCl/HA $(p=0.00005)$ or CAISU $(p=0.00096)$ extractions (Figure $2 \mathrm{E})$ and fewer glycoproteins and proteoglycans than any postdecellularization method (Figure 2E). When comparing individual fractions, the first fraction generated by the Gnd-HCl/HA method generates the greatest number of unique glycoprotein and proteoglycan peptides $(\mathrm{p}=0.023)$ (Figure 2E). Overall, utilizing a two-fraction Gnd-HCl/HA extraction provides significantly greater identification of collagen, proteoglycan, and glycoprotein peptides than all other tested methods.

ECM extraction methods which utilize urea have the potential to induce carbamylation at lysine and arginine residues, as well as peptide $\mathrm{N}$-termini. This modification can inhibit trypsin digestion and increase sample heterogeneity, convoluting data analysis and subsequent quantification. ${ }^{34}$ In our analysis, we identify averages of 763,641 , and 1523 carbamylated peptides per run in the CAISU, CAIS, and SCAD methods, respectively, compared to an average of 34 carbamylated peptides identified across the two fractions of the Gnd-HCl/HA method (Supporting Information Figure 5A). While the SCAD method induces more carbamylation than the other enzymatic extractions, all methods that utilize urea in an extraction buffer induce this modification extensively, likely contributing to deficits in ECM identification. On the other hand, hydroxylamine digest induces oxidation over other methods, where an average of 5954 oxidized peptides per run were identified in the $\mathrm{HA}$ fraction of the $\mathrm{Gnd}-\mathrm{HCl} / \mathrm{HA}$ method, compared to averages of 1702, 2290, and 3286 oxidized peptides in the CAISU, CAIS, and SCAD extracts, 
respectively (Supporting Information Figure 5B). While oxidation does not inhibit peptide cleavage, it can convolute analysis and protein quantification.

A
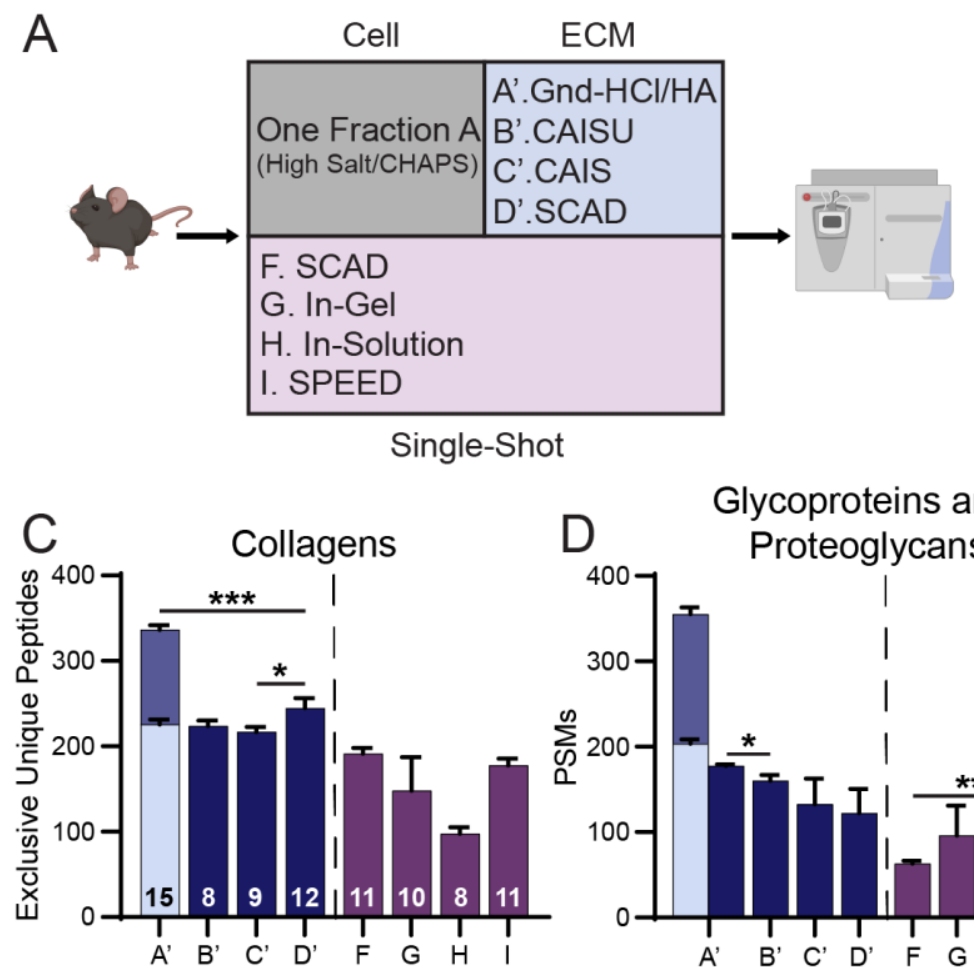

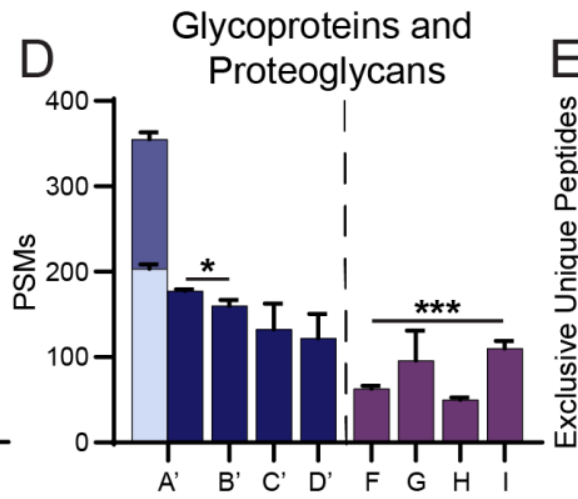

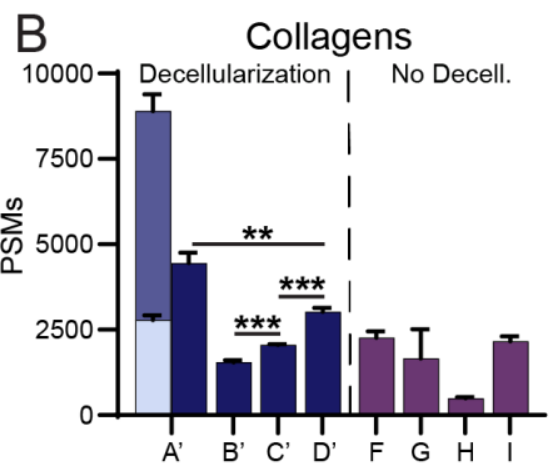

Glycoproteins and

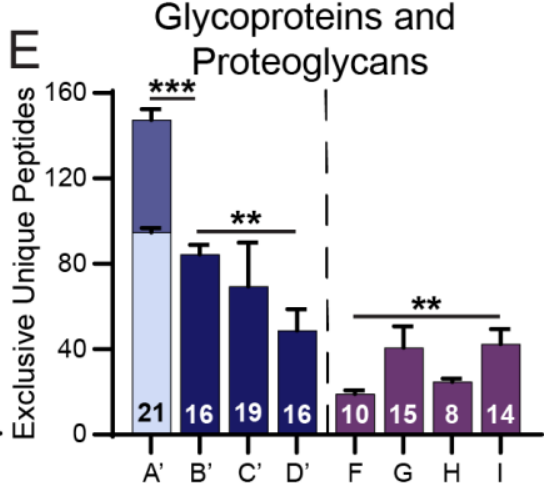

Figure 2. Effects of differing extracellular matrix extraction methods on uniform decellularized protein pellet. A) Workflow of ECM extraction (blue) and single-shot (purple) methods used. All ECM methods were preceded by the same decellularization. B) Total collagen PSMs identified by each method. Multi-step extraction (1') is shown as both a sum of the two fractions and the average PSMs per fraction. Plotted as average values with SD. For Gnd-HCl/HA method, light blue indicates $\mathrm{Gnd}-\mathrm{HCl}$ fraction, darker blue indicates HA fraction. C) Exclusive unique collagen peptides identified by each method. Numbers on bars represent the number of distinct collagen chains identified with a minimum of 2 peptides. D) Bar graph of glycoprotein and proteoglycan PSMs by each method. Multi-step extraction (1') shown as both a sum of two fractions and the average PSMs per fraction. E) Exclusive unique peptides identified for glycoproteins and proteoglycans by each method. Numbers on bars represent the number of glycoproteins and proteoglycans identified with a minimum of 2 peptides.

\section{ECM Extraction and Digestion Method Optimization}

The 2-fraction Gnd-HCl/HA extraction method was used for further optimization of ECM coverage, based on the above findings. Oxidative modifications observed with the use of hydroxylamine digestion ${ }^{4}$ can convolute data analysis and have the potential to reduce both the 
quantity and quality of protein identifications. On the other hand, if oxidation of Met residues is pushed toward completion without inducting other significant oxidations, this would have an advantageous effect on these metrics. In order to optimize this step, iterative changes to the hydroxylamine digestion protocol were explored with the goal of reducing unwanted modifications and ultimately increasing ECM protein identification. A reference protocol, as used in the previous section, was analyzed in parallel with each group of optimization conditions. Briefly, the reference protocol involves digestion with $1 \mathrm{M} \mathrm{HA}, \mathrm{pH} 9.0$ for 4 hours at $37^{\circ} \mathrm{C}$ with reduction and alkylation (R/A) prior to digestion. The previously published Gnd-HCl/HA method ${ }^{4}$ did not include R/A due to the lack of reducible disulfide bonds in collagen. However, R/A provided a $60 \%$ improvement in unique glycoprotein peptide identifications $(\mathrm{p}=0.0088)$, including a 10-fold increase in PSMs for fibrillin-1 (Supporting Information Figure 6) and improved overall CVs (Figure 3). Therefore, $\mathrm{R} / \mathrm{A}$ prior to HA digest was used as the reference method for the subsequent method optimization experiments. The second variable tested was pre-treatment of the pellet with $0.2 \% \mathrm{FA}$, as suggested by Bornstein and Bailan, ${ }^{35}$ which showed no significant benefit and increased the overall CV of ECM measurements (Figure 3).

We also evaluated the effects of changes in $\mathrm{pH}$, HA concentration, digestion time, and addition of an antioxidant (50mM methionine) on identification of ECM components. Most tested variations led to slight improvements in collagen PSMs but higher CVs and fewer total PSMs (Figure 3). Of note, $\mathrm{pH} 8.1$ with 50mM methionine improved the number of identified collagen PSMs. However, decreases in total and glycoprotein PSMs, as well as increased CV, make the method less suitable than the reference method unless maximizing total collagen PSMs is an objective of the analysis. In addition, 0.7M HA digestion for 16 hours performed significantly worse for all scoring metrics 
(Figure 3). This was consistent with previous findings where extended digestion times result in extensive peptide modification. ${ }^{4}$

$50 \mathrm{mM}$ methionine showed potential improvements in collagen PSMs and coverage over conditions without antioxidant. Therefore, the effects of more potent antioxidants including caffeic acid (CA), gallic acid (GA), and ascorbic acid (VitC) at varying concentrations on HA digestion were tested. All antioxidants showed significant improvement in sequence coverage of glycoproteins but not of proteoglycans or collagens (Figure 3). This is coupled with a significant drop in proteoglycan PSMs in comparison to the reference method. A final variable explored was deglycosylation, as improved identification of ECM glycoproteins and proteoglycans has been reported using PNGase F to remove N-linked glycans, ${ }^{20,36}$ as well as GAG-digesting enzymes chondroitinase $\mathrm{ABC}$ and heparinase $\mathrm{II} .^{36-38}$ The addition of PNGaseF slightly improved proteoglycan PSMs and sequence coverage over the reference method but also generated slight decreases in collagen PSMs and sequence coverage. PNGase F digestion significantly improved glycoprotein coverage $(\mathrm{p}=0.017)$ but did not improve glycoprotein PSMs (Figure 3 ). The addition of GAG removal enzymes (chondroitinase $\mathrm{ABC}$ and heparinase II) offered a significant improvement in proteoglycan coverage $(\mathrm{p}=0.012)$ but not PSMs. Improvement in proteoglycan identification was coupled with decreases in collagen $(p=0.0044)$ and glycoprotein $(p=0.0002)$ PSMs, causing GAG removal to result in overall worse ECM characterization than the reference method (Figure 3). When comparing deglycosylation (PNGase F) of both the Gnd-HCl and HA fractions, in general similar trends were observed when compared to the reference method for PSMs and sequence coverage of proteoglycans (Supporting Information Figure 7). However, improvement of proteoglycan coverage with the addition of PNGase F was highly significant $(p=0.00082)$ when the Gnd-HCl fraction was taken into account. 

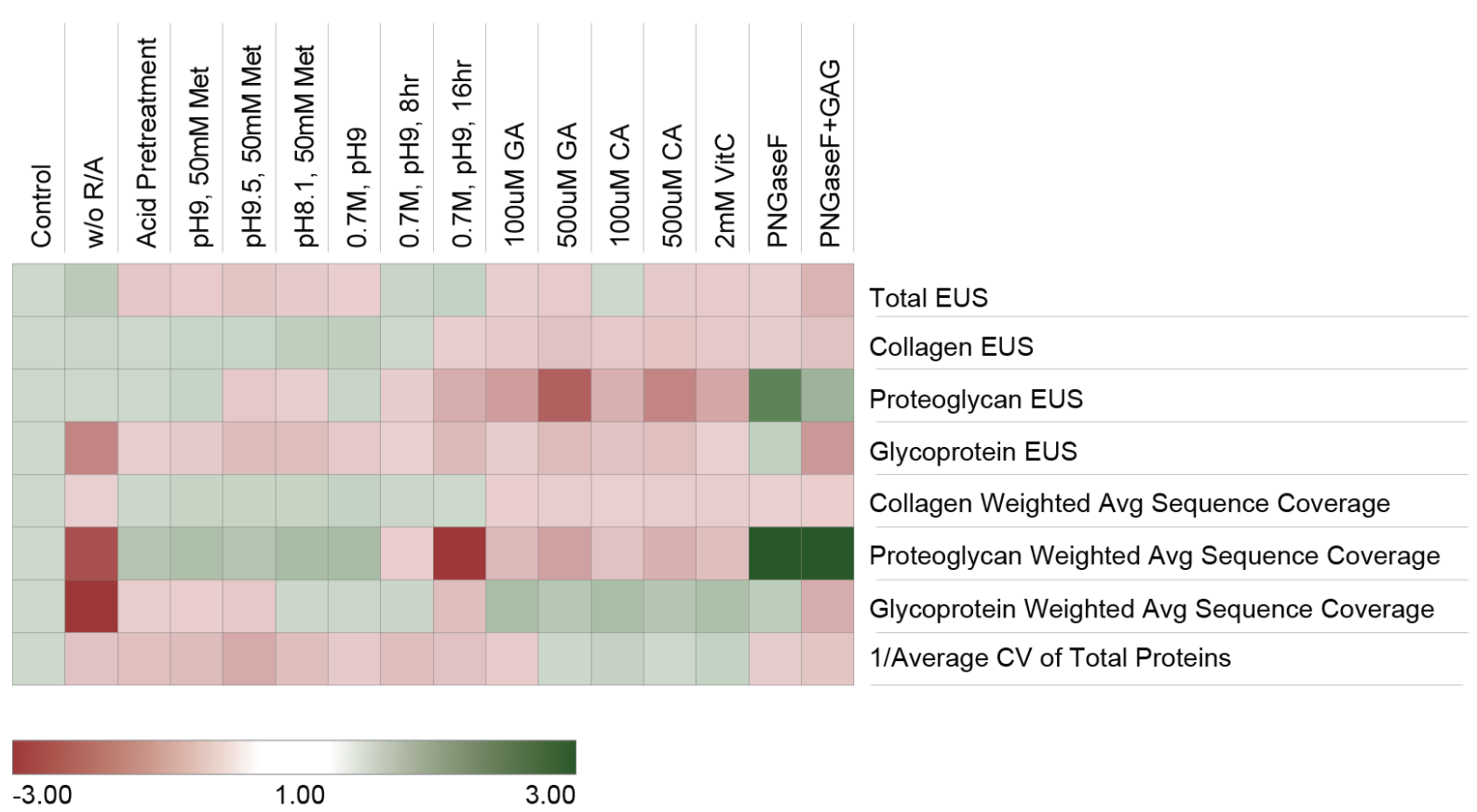

Figure 3. Optimization of Hydroxylamine (HA) digestion conditions. Hydroxylamine digest optimization was tested over several rounds of sample processing. Results were aggregated by comparing each digestion method to the reference sample run in parallel with treatment conditions (all $n=3$ ). Results are presented and colored as fold change in relation to reference. Reference HA digest was performed in $1 \mathrm{M} \mathrm{HA}-\mathrm{HCl} \mathrm{pH} 9.0$ at $37^{\circ} \mathrm{C}$ for $4 \mathrm{hrs}$ with reduction and alkylation prior to digestion. A detailed reference protocol can be found in the methods section. In the final column, GAG refers to the addition of GAG-digesting enzymes. Samples with fold change $<1$ are shown as negative reciprocal values.

\section{Evaluation of ECM Extraction and Digestion Methods on Isolated Organs}

All previous comparisons of ECM extraction methods were performed on an early generation

Orbitrap mass spectrometer (MS) due to the widespread use of this analytical platform and accessibility in core facilities. In order to perform more in-depth comparisons of ECM extraction methods and to determine if conclusions drawn from WMP hold true for organ specific analysis, extractions of lung, heart, kidney, and liver were analyzed on a modern, trapped ion mobility-time of flight MS system (Figure 4). Organs were chosen based on their widespread study in biomedical research and their varying ECM compositions. Organ samples were prepared using the 1-fraction A decellularization followed by the four tested ECM extraction methods. 
Consistent with our findings from WMP analysis, the Gnd-HCl/HA method provides more exclusive unique peptides for collagens in the lung (39\% greater), heart (38\% greater), kidney (26\% greater), and liver (46\% greater) than any other method (Figure 4A-D). Additionally, the Gnd-HCl/HA method performs similarly to, or better than, other methods in terms of glycoprotein and proteoglycan identification. The Gnd-HCl/HA method provided significantly more exclusive unique peptides than the next best method in lung $(11 \%$ greater, $\mathrm{p}=0.026)$ and heart $(32 \%$ greater, $\mathrm{p}=0.0004$ ) samples, but statistical significance was not reached in other organ comparisons (Figure 4E-H). Also consistent with results from WMP, the CAISU method performs better for identification of glycoproteins and proteoglycans across all organs than either the CAIS or SCAD extraction methods (Figure 4E-H).
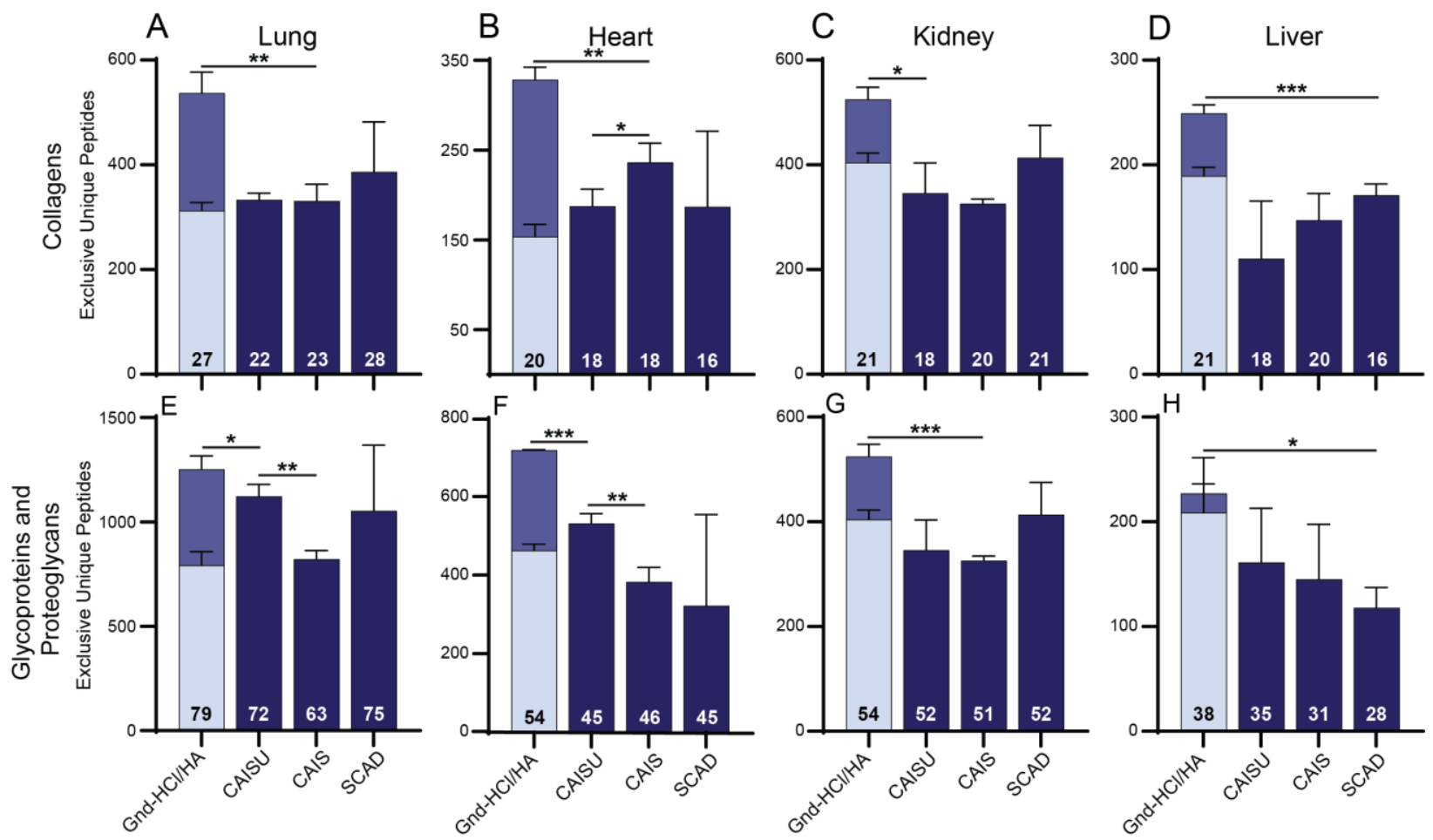

Figure 4. Effects of ECM extraction methods on uniformly decellularized mouse lung, heart, kidney, and liver. A-D) Total exclusive unique collagen peptides for each ECM extraction method. Numbers on bars represent the number of distinct collagen chains identified. For Gnd$\mathrm{HCl} / \mathrm{HA}$ method, light blue indicates $\mathrm{Gnd}-\mathrm{HCl}$ fraction, dark blue indicates new unique peptides from HA fraction. E-H) Total glycoprotein and proteoglycan exclusive unique peptides identified by each ECM extraction method. Numbers on bars represent the number of glycoproteins and 
proteoglycans identified. Bars represent the average of three replicates with error bars showing standard deviation (SD).

\section{$\underline{\text { Discussion }}$}

Based on the significant role that the structural matrix plays in shaping cellular phenotype $\mathrm{p}^{39,40}$ and, likewise, the influence of cell phenotype on stromal and matrix composition, ${ }^{41}$ optimized proteomic methods for ECM characterization are needed. In general, accurate tissue characterization requires analysis of the extracellular matrix, yet this class of proteins is underrepresented in tissue proteomic datasets. Several proteomic methods have been developed to improve characterization of the ECM over the last two decades. Key elements of these protocols involve 1) removal of cellular material to create an ECM enriched fraction, primarily using differential detergent extraction and 2) solubilization and efficient digestion of the resulting ECM. Various published decellularization methods were assessed for their ability to extract cellular proteins while leaving ECM proteins behind for analysis in subsequent fractions. The 1-fraction A and 4-fraction methods were found to remove more cellular proteins from the starting material than other tested methods. If cellular characterization and solubility profiling of cellular components are desired alongside ECM characterization, multi-fraction methods provide more cellular protein coverage when all fractions are analyzed. Also, of no surprise, single-shot methods result in fewer cellular PSMs than nearly all decellularization methods, with lower identifications due to the increased complexity and dynamic range of the extracted proteins, in part due to partial ECM extraction.

The largest amount of ECM proteins was extracted during decellularization using the 4-fraction method, evidenced by the high number of core matrisome protein and PSM identifications in the cellular fractions. Extraction of ECM proteins in the cellular fraction is undesirable due to difficulty detecting ECM proteins of interest against the background of abundant cellular protein. 
Additionally, when the 4-fraction method is used for decellularization prior to ECM extraction, the cellular fractions are often not analyzed via $\mathrm{MS},{ }^{20,42}$ causing all ECM proteins present in these fractions to be lost and ultimately resulting in quantitative error and potentially fewer identified core matrisome proteins within the ECM fractions. In our experience, to analyze these cellular fractions via LC-MS/MS additional detergent removal steps must be performed to avoid instrument contamination. Although the 1-fraction B method removes the least amount of ECM during decellularization, it does not efficiently extract cellular proteins, thus limiting subsequent ECM characterization.

After decellularization, the resulting pellets were processed using a single method for direct comparison of the resulting ECM fractions. Similar collagen PSMs were identified in the 1fraction A, 2-fraction, and 3-fraction methods, while the 4-fraction method resulted in significantly more collagen PSMs but fewer collagens and similar collagen sequence coverage to other methods. This is due to the high stringency of the 4-fraction decellularization method, extracting most noncollagen proteins in earlier fractions and allowing more sequencing time to be devoted to a subset of collagen peptides in the final ECM fraction. If optimal collagen signal is desired, performing 4fraction decellularization before ECM extraction can provide greater collagen spectral matches but not necessarily higher sequence coverage of collagen proteins. Additionally, the 4-fraction method generates significantly less sequence coverage of ECM glycoproteins and proteoglycans, hampering its overall characterization of core matrisome proteins. The 2-fraction decellularization method, on the other hand, provided the greatest coverage of glycoproteins and proteoglycans within the ECM fractions, making this the method of choice when these protein classes are a priority. 
Total PSMs, ECM PSMs, and method precision have been discussed above. These evaluation metrics along with time, ease, and MS compatibility are shown in Table 2. Each category was ranked on a scale of 1-10 with 1 being the lowest possible score and 10 being optimal (ranking criteria are further defined in methods). MS compatibility was ranked based on the use of MSincompatible detergents throughout the course of the method. Acetone precipitation or a detergent removal column can be utilized within any protocol to reduce risk of MS contamination, but these steps can generate additional cost, variability, and protein loss. Methods that utilize SDS and NP40, such as the 3-fraction method, can cause residual contamination during MS analysis of subsequent ECM fractions and require additional pellet washing or acetone precipitation of the ECM fractions. The ease criterion is a ranking of the relative ease of each method in comparison to the other protocols, considering the number of processing steps and potential for increased analytical $\mathrm{CV}$. The 4-fraction method displayed higher variability than other methods across two triplicate rounds, likely due to the large number of buffer exchanges required during processing (10) compared to other methods. As this table shows, there is not one method that is superior in all categories. Instead, a method should be selected specifically based on project aims, constraints, and proteins of interest.

Table 2. Comparison of decellularization methods for ECM enrichment. Decellularization (green) and single-shot (purple) methods are ranked in each category from one to ten - one being totally insufficient and ten being optimal. Total score is calculated as a weighted average of all assessed categories (described in methods).

\begin{tabular}{|c|c|c|c|c|c|c|c|}
\hline Method & Time & Ease & MS Compatability & Total Protein IDs & Precision & $\begin{array}{c}\text { ECM in Cell } \\
\text { Fraction }\end{array}$ & Total Score \\
\hline \hline 1-Fraction A & 8 & 9 & 7 & 8 & 9 & 8 & 82 \\
\hline 1-Fraction B & 3 & 9 & 6 & 7 & 8 & 9 & 74 \\
\hline 2-Fraction & 9 & 8 & 6 & 8 & 9 & 7 & 79 \\
\hline 3-Fraction & 8 & 7 & 6 & 9 & 8 & 7 & 77 \\
\hline 4-Fraction & 6 & 6 & 6 & 9 & 6 & 6 & 68 \\
\hline SCAD & 6 & 8 & 8 & 6 & 8 & N/A & 71 \\
\hline In-Gel & 7 & 7 & 8 & 7 & 6 & N/A & 69 \\
\hline In-Solution & 9 & 9 & 9 & 5 & 9 & N/A & 78 \\
\hline SPEED & 9 & 7 & 9 & 7 & 9 & N/A & 81 \\
\hline
\end{tabular}


The second key element of an effective ECM proteomic approach is efficient digestion of matrix proteins. This task is hampered by the relative protease resistance of the ECM. Four published ECM extraction methods were evaluated on both WMP and isolated organ samples following uniform decellularization. While it is impractical to test all published methods and extensively test all variables, these four methods cover the major ECM-based methods that are currently used. As stated above, the two-step Gnd-HCl/HA method identified the greatest number of proteins, PSMs, and unique peptides from all core matrisome categories in analysis of both WMP and isolated organs. While the $\mathrm{Gnd}-\mathrm{HCl}$ fraction of the $\mathrm{Gnd}-\mathrm{HCl} / \mathrm{HA}$ extraction alone performs similarly to other tested methods, the following HA digest provides 355 additional unique core matrisome peptides on average across analyzed organs, leading the 2-fraction HA digest method to provide improved core matrisome characterization over other tested methods. This, combined with the higher sequence coverage of collagens, glycoproteins, and proteoglycans, makes the Gnd-HCl/HA extraction the recommended method for obtaining more in-depth coverage of the ECM, despite the additional time it requires. The proportion of ECM peptides which are uniquely identified by the HA digest in the Gnd-HCl/HA method varies greatly between tested organs, with $40 \%$ of ECM peptides uniquely identified in the HA fraction of heart samples but only $15 \%$ in the HA fraction of kidney. These differences reflect varying relative ECM protein abundance, association, and crosslinking - attributes which are often altered during development and disease progression.

Of the enzymatic ECM extractions, SCAD after decellularization provides a greater number of identified collagens and more unique collagen peptides than the CAIS or CAISU methods in WMP analysis. The CAISU method, on the other hand, yielded more unique glycoprotein and proteoglycan peptide identifications and greater sequence coverage than other enzymatic methods 
in all samples. Therefore, enzymatic methods should be chosen depending on the ECM protein categories of interest when only one ECM fraction is analyzed.

When comparing faster, single-shot methods, SCAD and SPEED generated significantly higher PSMs for collagens than the CAISU method, even though these methods lack decellularization steps to remove cellular contaminants. SPEED also provides the greatest number of unique peptides from glycoproteins and proteoglycans of the tested single-shot methods. SCAD performs poorly in this matrisome category, revealing that SPEED is the best performing single-shot method for overall ECM coverage. SPEED is a favorable alternative to longer ECM extractions that provides good ECM coverage and unique peptide IDs when higher throughput ECM analysis is desired, and multiplexing is not available or otherwise used.

A condensed evaluation of all ECM extraction and single-shot methods can be found in Table 3 . Some of the criteria for evaluation have already been discussed above. This table also addresses the time needed to perform each extraction and the relative ease of each protocol. There is no one perfect choice for an ECM extraction method but these evaluation metrics should help guide researchers to a method of choice for a given set of objectives.

Table 3. Comparison of ECM extraction/digestion methods. ECM extraction (blue) and singleshot (purple) methods are ranked in each category from one to ten - with one being totally insufficient and ten being optimal. Total score is calculated as a weighted average of all assessed categories (described in methods).

\begin{tabular}{|c|c|c|c|c|c|c|}
\hline Method & Time & Ease & Collagens & $\begin{array}{c}\text { Glycoproteins and } \\
\text { Proteoglycans }\end{array}$ & Precision & Total Score \\
\hline \hline Gnd-HCl/HA & 5 & 6 & 9 & 9 & 8 & 81 \\
\hline CAISU & 8 & 7 & 6 & 8 & 9 & 75 \\
\hline CAIS & 6 & 8 & 7 & 7 & 7 & 70 \\
\hline SCAD + Decell. & 5 & 8 & 8 & 6 & 7 & 69 \\
\hline SCAD & 5 & 8 & 6 & 3 & 8 & 56 \\
\hline In-Gel & 7 & 7 & 5 & 5 & 5 & 54 \\
\hline In-Solution & 9 & 9 & 4 & 4 & 8 & 58 \\
\hline SPEED & 9 & 7 & 6 & 6 & 7 & 66 \\
\hline
\end{tabular}


Based on the favorable results obtained using the two-step Gnd-HCl/HA method, we performed comparisons aimed to improve upon this method. Reduction and alkylation prior to hydroxylamine digestion was the first change tested. Based on superior initial results, HA digestion with prior reduction and alkylation became the reference method for subsequent optimization. Most tested variables resulted in both improved and reduced individual ECM category performance. Given the fact that most alterations added an additional cost or step, which increases potential variability, for most projects the published HA digestion method $^{4}$ with added reduction and alkylation prior to HA digestion is recommended. The one exception is the addition of PNGaseF for proteoglycan coverage. Somewhat surprisingly, there is a clear increase in proteoglycan PSMs upon addition of PNGaseF but no improvement in identification of collagens or ECM glycoproteins. It is important to note that the use of PNGaseF adds extra cost to each sample preparation. In addition, the improvement in proteoglycan coverage but not PSMs suggests that some extracted glycopeptides remained unidentified by database searching until deglycosylated by PNGaseF. The addition of PNGaseF could be a worthwhile investment if optimizing proteoglycan coverage is a priority.

The first set of analysis was performed using an older generation orbitrap instrument that is likely to be encountered by many groups interested in the analysis of ECM through collaboration or core facilities. While this analytical platform offers high mass accuracy and resolution which are valuable for reducing false positives, the sensitivity and scan speed does not match more modern instrumentation. Additionally, WMP was used for initial testing due to the high complexity of the sample, allowing for comparison of methods without biasing results toward a specific application. However, proteomics studies are generally performed using isolated tissue samples, their substructures, or micro-dissected regions. As a result, we also evaluated the chosen ECM extraction methods on isolated organs using a modern timsTOF mass spectrometer to assess 
method performance on real-world samples using a state-of-the-art instrument. In general, comparisons between methods revealed during WMP testing were consistent with those observed in analysis of individual organs. While method comparisons are mostly consistent across the tested organs, further method optimization may be required to obtain high-quality ECM characterization for organs with very unique compositional profiles (e.g. bone). Here, we evaluated extraction and digestion of tissues in a non-limiting sample regime (1-5 mg dry weight). However, we have also had success applying our ECM extraction method to human tissue samples $(<10 \mu \mathrm{g})$ from lasercapture microdissection (LCM) extraction (Supporting Information Figure 8) with good ECM coverage, extending these techniques for spatial analysis of the ECM.

Of the over 200 proteins defined as "core matrisome" within the MatrisomeDB, ${ }^{28} 138$ were identified across the 4 analyzed organs. Within the ECM, collagen is both highly abundant and highly modified. Increased fractionation of ECM samples may reveal differences in identification of low-abundance ECM proteins between methods which were not addressed in this study. All data was acquired using data-dependent acquisition because it is the most accessible acquisition method and does not require generation of a spectral library to achieve high-quality results. However, this allows for stochastic sampling of low abundance precursor species and generates more missing values. This limitation could be diminished by using data-independent acquisition, as has been previously demonstrated for ECM analysis. ${ }^{43}$ However, the high complexity of posttranslational modifications commonly encountered on ECM peptides (more than 5 modifications per peptide with various positional isomers) presents a significant, largely unresolved issue with search routines. This also presents unique opportunities for the use of ion mobility for further resolution and characterization of these complex peptide species. 
The choice of decellularization method should be based on the objectives of a given project. If minimizing sample processing time and ECM extraction are priorities, the 1 fraction A method is recommended. However, if deeper proteome coverage or solubility profiling of cellular components is desired, the 2-fraction or 3-fraction decellularization methods with analysis of all produced fractions are recommended. Deeper cellular coverage could also be provided by performing the 1 fraction A decellularization followed by offline fractionation prior to MS analysis with less potential for variability compared to these methods. For optimal ECM coverage, the Gnd$\mathrm{HCl} / \mathrm{HA}$ extraction protocol is recommended as it produces the greatest number of PSMs and highest sequence coverage of core matrisome proteins. Disease progression and aging have been shown to increase the resistance of the insoluble ECM to extraction, ${ }^{44}$ increasing the necessity of effectively extracting the insoluble ECM via chemical digestion. Additionally, the Gnd-HCl/HA extraction produces two separate ECM fractions which can be analyzed independently, further increasing ECM identifications and allowing for assessment of ECM solubility. Alterations in protein solubility can provide important information regarding disease progression ${ }^{45,46}$ which cannot be derived from single-fraction abundance measurements alone. Therefore, the Gnd$\mathrm{HCl} / \mathrm{HA}$ method is the method of choice for optimized ECM analysis. For a single-shot method, the SPEED method is the method of choice due to its ability to provide moderately high coverage of both core matrisome and cellular components using a single MS run. However, throughput can also be increased using multiplexing reagents and faster acquisition times available on modern MS instrumentation.

Mass spectrometry has increasingly played a central role in biomolecular characterization of tissues for the study of development, health, and disease. While the instrumentation, data acquisition routines, and bioinformatic tools facilitating proteomics workflows are consistently 
improving, less attention has been given to improving sample preparation methods and ensuring that all relevant material from a sample is analyzed. Without optimized sample preparation methods, a significant portion of the tissue proteome will be missed. ECM proteins will remain insoluble and studies will not achieve characterization of a protein fraction that is largely responsible for the underlying cell phenotype and biomechanics of a tissue. The methods evaluated and optimized here should help facilitate studies of tissue microenvironments in development, disease progression and aging.

\section{ASSOCIATED CONTENT.}

\section{Supporting Information.}

The following files are available.

Supplementary Figures (.docx)

Supplementary Data - Exclusive Unique Peptides

(SupplementaryData_ExclusiveUniquePeptides.xlsx)

Supplementary Data - Total Peptide Spectral Matches

(SupplementaryData_TotalPeptideSpectralMatches.xlsx)

The mass spectrometry proteomics data have been deposited to the ProteomeXchange Consortium via the MassIVE partner repository with the data set identifier PXD021778. Data available upon request - contact corresponding author for access.

\section{AUTHOR INFORMATION}

\section{Corresponding Author}

Email: kirk.hansen@cuanschutz.edu 


\section{Author Contributions}

The manuscript was written through contributions of all authors. All authors have given approval to the final version of the manuscript. $\$ \mathrm{MCM}$ and LRS contributed equally.

\section{Funding Sources}

This work was supported by the NIH (Grant No. R33CA183685, RM1GM131968, P01HL152961, R01HL146519) and the Cancer Center Support Grant (P30CA046934) and the Lung Foundation Netherlands (Longfonds, 6.1.15.017).

\section{ACKNOWLEDGMENT.}

We would like to thank the numerous students, technicians and collaborators that have evaluated, utilized, and provided feedback on these methods throughout the years. Workflow figures throughout the publication were created with BioRender.com.

\section{ABBREVIATIONS}

$\underline{\text { Abbreviation }} \underline{\text { Full name }}$

ABC Ammonium bicarbonate

ACN Acetonitrile

CA Caffeic acid

CAIS Chaotrope-assisted in-solution digest

CAISU Chaotrope-assisted in-solution digest with ultrasonication

CHAPS 3-[(3-cholamidopropyl)dimethylammonio]-1-propanesulfonate

$\mathrm{CV} \quad$ Coefficient of variance

DOC Sodium deoxycholate

DTT Dithiothreitol

ECM Extracellular matrix

EDTA Ethylenediaminetetraacetic acid 


\begin{tabular}{|c|c|}
\hline FA & Formic acid \\
\hline FDR & False discovery rate \\
\hline GA & Gallic acid \\
\hline GAG & Glycosaminoglycan \\
\hline Gnd-HCl & Guanidine hydrochloride \\
\hline HA & Hydroxylamine hydrochloride \\
\hline HEPES & 4-(2-hydroxyethyl)-1-piperazineethanesulfonic acid \\
\hline IAM & Iodoacetamide \\
\hline $\mathrm{K} 2 \mathrm{CO} 3$ & Potassium carbonate \\
\hline $\mathrm{KCl}$ & Potassium chloride \\
\hline LC-MS/MS & Liquid chromatography tandem mass spectrometry \\
\hline $\mathrm{MgCl}_{2}$ & Magnesium chloride \\
\hline MS & Mass spectrometry \\
\hline $\mathrm{NaCl}$ & Sodium chloride \\
\hline $\mathrm{NaOV}$ & Sodium orthovanadate \\
\hline NP-40 & Nonidet-P40 \\
\hline PI & Protease Inhibitor \\
\hline Pipes & Piperazine-N,N'-bis(2-ethanesulfonic acid) \\
\hline PSM & Peptide spectral match \\
\hline $\mathrm{R} / \mathrm{A}$ & Reduction and alkylation \\
\hline SCAD & Surfactant and chaotropic agent assisted sequential extraction/on-pellet digestion \\
\hline SDS & Sodium dodecyl sulfate \\
\hline SPEED & Sample preparation by easy extraction and digestion \\
\hline TFA & Trifluoroacetic acid \\
\hline Tris-HCl & Tris(hydroxymethyl)aminomethane hydrochloride \\
\hline VitC & Ascorbic acid \\
\hline WMP & Whole mouse powder \\
\hline
\end{tabular}




\section{REFERENCES}

1. Hynes, R. O. Extracellular matrix: not just pretty fibrils. Science (80-. ). 326, 1216-1219 (2009).

2. Yurchenco, P. D., Birk, D. E. \& Mecham, R. P. Extracellular Matrix Assembly and Structure. (Academic press, 1994).

3. Frantz, C., Stewart, K. M. \& Weaver, V. M. The extracellular matrix at a glance. J. Cell Sci. 123, 4195-4200 (2010).

4. Barrett, A. S. et al. Hydroxylamine chemical digestion for insoluble extracellular matrix characterization. J Proteome Res. 44, 319-335 (2017).

5. Stoilov, I., Starcher, B. C., Mecham, R. P. \& Broekelmann, T. J. Measurement of elastin, collagen, and total protein levels in tissues. Methods in Extracellular Matrix Biology 143, (Elsevier Inc., 2018).

6. Hon, W. C. et al. Structural basis for the recognition of hydroxyproline in HIF-1 $\alpha$ by pVHL. Nature 417, 975-978 (2002).

7. Schmelzer, C. E. H. et al. Prolyl hydroxylation in elastin is not random. Biochim. Biophys. Acta - Gen. Subj. 1860, 2169-2177 (2016).

8. Weiskirchen, S., Tag, C. G., Sauer-lehnen, S., Tacke, F. \& Weiskirchen, R. Probing Collagen Organization: Practical Guide for Second- Harmonic Generation (SHG) Imaging. 1627, 165-191 (2017).

9. Mostaço-Guidolin, L., Rosin, N. L. \& Hackett, T. L. Imaging collagen in scar tissue: Developments in second harmonic generation microscopy for biomedical applications. Int. J. Mol. Sci. 18, (2017).

10. Kim, M.-S. et al. A draft map of the human proteome. Nature 509, 575-581 (2014).

11. Schmidt, T. et al. ProteomicsDB. Nucleic Acids Res. 46, D1271-D1281 (2018). 
12. Uhlén, M. et al. Tissue-based map of the human proteome. Science (80-. ). 347, (2015).

13. Wilhelm, M. et al. Mass-spectrometry-based draft of the human proteome. Nature 509, 582-587 (2014).

14. Hill, R. C., Calle, E. A., Dzieciatkowska, M., Niklason, L. E. \& Hansen, K. C. Quantification of Extracellular Matrix Proteins from a Rat Lung Scaffold to Provide a Molecular Readout for Tissue Engineering. Mol. Cell. Proteomics 14, 961-973 (2015).

15. Didangelos, A. et al. Proteomics characterization of extracellular space components in the human aorta. Mol. Cell. Proteomics 9, 2048-2062 (2010).

16. Lennon, R. et al. Global analysis reveals the complexity of the human glomerular extracellular matrix. J. Am. Soc. Nephrol. 25, 939-951 (2014).

17. Wilson, R. et al. Changes in the chondrocyte and extracellular matrix proteome during postnatal mouse cartilage development. Mol. Cell. Proteomics 11, 1-18 (2012).

18. Brown, K. A. et al. High-Throughput Proteomics Enabled by a Photocleavable Surfactant. Angew. Chemie 132, 8484-8488 (2020).

19. Schiller, H. B. et al. Time- and compartment-resolved proteome profiling of the extracellular niche in lung injury and repair. Mol. Syst. Biol. 11, 1-19 (2015).

20. Naba, A., Clauser, K. R. \& Hynes, R. O. Enrichment of Extracellular Matrix Proteins from Tissues and Digestion into Peptides for Mass Spectrometry Analysis. J. Vis. Exp. (2015). doi:10.3791/53057

21. Ma, F., Liu, F., Xu, W. \& Li, L. Surfactant and Chaotropic Agent Assisted Sequential Extraction/On-Pellet Digestion (SCAD) for Enhanced Proteomics. J Proteome Res. 17, 2744-2754 (2015).

22. Hansen, K. C. et al. An In-solution Ultrasonication-assisted Digestion Method for Improved Extracellular Matrix Proteome Coverage. Mol. Cell. Proteomics 8, 1648-1657 (2009).

23. Decaris, M. L. et al. Proteomic analysis of altered extracellular matrix turnover in 
bleomycin-induced pulmonary fibrosis. Mol. Cell. Proteomics 13, 1741-1752 (2014).

24. Önnerfjord, P., Khabut, A., Reinholt, F. P., Svensson, O. \& Heinegård, D. Quantitative proteomic analysis of eight cartilaginous tissues reveals characteristic differences as well as similarities between subgroups. J. Biol. Chem. 287, 18913-18924 (2012).

25. Gluck, J. M. et al. Biochemical and biomechanical properties of the pacemaking sinoatrial node extracellular matrix are distinct from contractile left ventricular matrix. PLoS One 12, (2017).

26. Epstein, E. H. \& Munderloh, N. H. Isolation and characterization of CNBr peptides of human $[\alpha 1(\mathrm{III})] 3$ collagen and tissue distribution of $[\alpha 1(\mathrm{I})] 2 \alpha 2$ and $[\alpha 1(\mathrm{III})] 3$ collagens. $J$. Biol. Chem. 250, 9304-9312 (1975).

27. Krasny, L. et al. Comparative proteomic assessment of matrisome enrichment methodologies. Biochem. J. 473, 3979-3995 (2016).

28. Shao, X., Taha, I. N., Clauser, K. R., Gao, Y. (Tom) \& Naba, A. MatrisomeDB: The ECMprotein knowledge database. Nucleic Acids Res. 48, D1136-D1144 (2020).

29. $\mathrm{Xu}, \mathrm{H}$. et al. Comparison of Decellularization Protocols for Preparing a Decellularized Porcine Annulus Fibrosus Scaffold. PLoS One 9, (2014).

30. Doellinger, J., Schneider, A., Hoeller, M. \& Lasch, P. Sample Preparation by Easy Extraction and Digestion (SPEED) - A Universal, Rapid, and Detergent-free Protocol for Proteomics Based on Acid Extraction. Mol Cell Proteomics 19, 209-222 (2019).

31. Makridakis, M. \& Vlahou, A. GeLC-MS: A Sample Preparation Method for Proteomics Analysis of Minimal Amount of Tissue. Methods Mol. Biol. 1788, 165-175 (2018).

32. Wiśniewski, J. R., Zougman, A., Nagaraj, N. \& Mann, M. Universal sample preparation method for proteome analysis. Nat. Methods 6, 359-362 (2009).

33. Mellacheruvu, D. et al. The CRAPome: A contaminant repository for affinity purificationmass spectrometry data. Nat. Methods 10, 730-736 (2013). 
34. Sun, S., Zhou, J.-Y., Yang, W. \& Zhang, H. Inhibition of Protein Carbamylation in Urea Solution Using Ammonium Containing Buffers. Anal Biochem. 76-81 (2014). doi:10.1021/n1061786n.Core-Shell

35. Bornstein, P. \& Balian, G. Cleavage at Asn-Gly Bonds with Hydroxylamine. Methods Enzym. 47, 132-45 (1977).

36. Barallobre-barreiro, J., Baig, F., Fava, M., Yin, X. \& Mayr, M. Glycoproteomics of the Extracellular Matrix: A Method for Intact Glycopeptide Analysis Using Mass Spectrometry. J. Vis. Exp. 122, 1-10 (2017).

37. Raghunathan, R., Sethi, M. K. \& Zaia, J. On-slide tissue digestion for mass spectrometry based glycomic and proteomic profiling. MethodsX 6, 2329-2347 (2019).

38. Klein, J. A., Meng, L. \& Zaia, J. Deep sequencing of complex proteoglycans: A novel strategy for high coverage and sitespecific identification of glycosaminoglycanlinked peptides. Mol. Cell. Proteomics 17, 1578-1590 (2018).

39. Kumar, A., Placone, J. K. \& Engler, A. J. Understanding the extracellular forces that determine cell fate and maintenance. Co. Biol. 144, 4261-4270 (2017).

40. Muncie, J. M. \& Weaver, V. M. The Physical and Biochemical Properties of the Extracellular Matrix Regulate Cell Fate. Curr Top Dev Biol. 130, 1-37 (2018).

41. Malik, R., Lelkes, P. I. \& Cukierman, E. BIOMECHANICAL and BIOCHEMICAL REMODELING of STROMAL EXTRACELLULAR MATRIX IN CANCER. Trends Biotechnol. 33, 230-236 (2015).

42. Hynes, R. O. \& Naba, A. Overview of the matrisome-An inventory of extracellular matrix constituents and functions. Cold Spring Harb. Perspect. Biol. 4, 1-16 (2012).

43. Krasny, L. et al. SWATH mass spectrometry as a tool for quantitative profiling of the matrisome. J. Proteomics 189, 11-22 (2018).

44. McCabe, M. C. et al. Alterations in Extracellular Matrix Composition during Aging and 
Photoaging of the Skin. Matrix Biol. Plus (2020).

45. Vecchi, G. et al. Proteome-wide observation of the phenomenon of life on the edge of solubility. Proc. Natl. Acad. Sci. U. S. A. 117, 1015-1020 (2020).

46. Pace, M. C. et al. Changes in proteome solubility indicate widespread proteostatic disruption in mouse models of neurodegenerative disease. Acta Neuropathol. 136, 919-938 (2018). 